\title{
High-Resolution Weather Database for the Terminal Area of Frankfurt Airport
}

\author{
Michael Frech, Frank Holzäpfel, Arnold Tafferner, and Thomas Gerz \\ Institut für Physik der Atmosphäre, DLR, Oberpfaffenhofen, Germany
}

(Manuscript received 12 June 2006, in final form 25 January 2007)

\begin{abstract}
A 1-yr meteorological dataset for the terminal area of Frankfurt Airport in Germany has been generated with a numerical weather prediction system to provide a synthetic though realistic database for the evaluation of new operational aircraft arrival procedures and their associated risks. The comparison of the 1-yr dataset with a local surface wind climatology indicates that the main climatological features are recovered. A subset of 40 days is validated against measurements from a sound detection and range/radio acoustic sounding system (SODAR/RASS) taken at Frankfurt Airport. The RMS errors of wind speed and direction are between $1.5 \mathrm{~m} \mathrm{~s}^{-1}$ at the surface and $2 \mathrm{~m} \mathrm{~s}^{-1}$ at $300 \mathrm{~m}$ and $40^{\circ}$, respectively. The frequency distribution of meteorological parameters, such as the wind component perpendicular to the glide path, shear, and thermal stratification, show good agreement with observations. The magnitude of the turbulent energy dissipation rate near the surface is systematically overestimated, whereas above $100 \mathrm{~m}$ the authors find on average a slight underestimation. The analysis of the database with respect to crosswind conditions along the glide path indicates only a time fraction of $12 \%$ for which the crosswind is above a threshold of $2 \mathrm{~m} \mathrm{~s}^{-1}$. A similar result is obtained using a grid point near the airport that mimics a wind profiler, which suggests that in a majority of cases a wind profiler appears sufficient to cover the expected crosswind conditions along the glide path. A simple parameterization to account for the crosswind variability along the glide path is proposed.
\end{abstract}

\section{Introduction}

Airport demand traffic is expected to more than double within the next 10-20 yr (Eurocontrol 2004). Already today, major airports operate close to their capacity limits. Since airport expansions are often not possible, other means to increase capacity are needed. One option is to reduce the separation of approaching aircraft. Today, fixed separations, which were established in the 1970s when Boeing introduced their B747, are required between consecutive aircraft depending on the weight class of the leading and following aircraft to avoid hazardous wake vortex encounters. The strength of a wake vortex is governed by the weight, speed, and span of the aircraft and air density. In case of an encounter, an aircraft experiences a rolling moment that may be hazardous, especially if an aircraft is close to the surface during landing or departure.

Corresponding author address: Dr. Michael Frech, Deutscher Wetterdienst, Messsysteme, Albin-Schwaiger-Weg 10, D-82383 Hohenpeissenberg, Germany.

E-mail: michael.frech@dwd.de

DOI: 10.1175/2007JAMC1513.1

(C) 2007 American Meteorological Society
In day-to-day operations, separations are already reduced under visual meteorological conditions (VMCs). If the pilot of a following aircraft can see the leading aircraft, air traffic control (ATC) often offers the pilot the option to reduce separation under his responsibility. This procedure only assumes a simplistic rule of thumb. Normally wake vortices descend below the glide path because of mutual velocity induction and are advected laterally by crosswind, so that pilots try to fly above and upwind of the leading aircraft where practicable. The relatively small number of known serious encounters seems to support this procedure. However, the recent investigation of flight data recordings clearly indicated that encounters happen in reality more frequently than previously thought (de Bruin et al. 2003). Wake vortices may persist up to $5 \mathrm{~min}$ and have not fully dissipated under existing separations (Frech and Zinner 2004). Most of the time the glide path is cleared from wake vortices through the lateral transport by crosswind and the mutually induced vertical descent of the vortex pair itself. These appear to be the main hazard reduction mechanisms, while atmospheric turbulence and stable stratification in addition contribute to 
an accelerated wake vortex decay. Most of the encounters are obviously not hazardous and sometimes they are not recognized as such by pilots, who may interpret an encounter as atmospheric turbulence. This is related to the fact that the two counterrotating vortex tubes typically are deformed and partially decay while they evolve in the atmosphere. This is accompanied with a loss of coherence. The rate at which a wake vortex deforms and decays is mainly dependent on the atmospheric state. Therefore an aircraft may encounter only small portions of a wake, which then is perceived just as turbulence. The wake vortex interaction with the atmosphere may also lead to a rebound under strong stable situations and shear (Holzäpfel et al. 2001; Holzäpfel 2005). In particular, the interaction with shear is complex and very much dependent on the shape of the wind profile.

The aforementioned meteorological parameters have to be known all along the glide path or departure corridor at a high spatial and temporal resolution. Considering the typical time and length scale of a wake vortex, the vertical resolution should be on the order of $50 \mathrm{~m}$ or higher and the temporal resolution on the order of 2 min. In principle, the required resolution can be realized with existing sensor technology. However, a substantial effort is needed to guarantee a reliable temporal and spatial data coverage along the glide path. Since May 2004, a wind and temperature profiler is operated at Frankfurt Airport by the German air navigation service provider (DFS; Konopka and Fischer 2005). However, further long-term measurements of meteorological conditions at high temporal and spatial resolution (which, e.g., cover the glide path) do not exist for the Frankfurt terminal area.

A number of projects have been launched in order to develop new technologies and procedures to increase capacity at an airport (see, e.g., Gerz et al. 2005). However, before changing an existing system, a risk assessment is mandatory according to the safety regulations developed at Eurocontrol. The procedure is described in the European Safety Regulatory Requirement (ESARR 4; Eurocontrol 2001), which deals with risk assessment and mitigation in air traffic management. A risk assessment must consider all parameters that may affect safety of a new procedure or system. Meteorological conditions are crucial for wake vortex decay and transport and have to be represented adequately in a risk assessment. For this purpose, a 1-yr meteorological database for the Frankfurt terminal area has been generated using the nowcasting system named Nowcasting Wake Vortex Impact Variables (NOWVIV; Gerz et al. 2005). This 1-yr dataset comprises typical weather conditions and includes already typical features of a long- term surface wind climatology. This dataset allows one to test new operational concepts with realistic meteorological input and to investigate potential benefits and associated risks.

The approach to compute a surface wind climatology for a specific airport by using a mesoscale model is presented by Heimann (1986). To minimize the computational effort, the model was initialized using classes of geostrophic wind and temperature profiles that were analyzed from reanalysis data. Comparable approaches to estimate wind climatologies for wind power applications at specfic locations are shown in, for example, Bergström (1996), Sandström (1997), and Mengelkamp (1999). The aforementioned studies mainly focus on the mean climatological wind conditions and not on the temporal and spatial variability of everyday weather in a specific area. However, the consideration of weather variability is an important aspect in the context of assessing wake vortex hazards in the airport terminal area.

In this study, a subset of the 1-yr database is analyzed in more detail for a period of 40 days when a dedicated wake measurement campaign was carried out at Frankfurt Airport. During this measurement campaign, a sound detection and range/radio acoustic sounding system (SODAR/RASS) and a lidar provided profile measurements of meteorological variables. These data are used to analyze the quality of the predicted meteorological parameters, which affects wake transport and decay.

We first introduce the NOWVIV system and the setup for the 1-yr simulation. In section 3 we compare the frequency distribution of surface wind speed and direction derived from the database with a 30 -yr surface wind climatology. In section 4, we compare the NOWVIV results with measurements of wind and temperature taken during a field campaign at Frankfurt Airport. We then investigate aspects of the 1-yr database that are of interest for operational application.

\section{Production and description of the database}

NOWVIV has been successfully employed for predictions of wake vortex environmental parameters in the field campaigns WakeOP 2001 and WakeTOUL 2002 of the projects Wirbelschleppe and C-Wake and in the measurement campaign at Frankfurt Airport accomplished in autumn 2004 (Gerz et al. 2005). The core of NOWVIV is the mesoscale model fifth-generation Pennsylvania State University-National Center for Atmospheric Research Mesoscale Model (MM5; Grell et al. 1994, 2000), where a second-order level-3 turbulence closure scheme (Burk and Thompson 1989) is em- 
$\mathrm{Z}(\mathrm{m})$

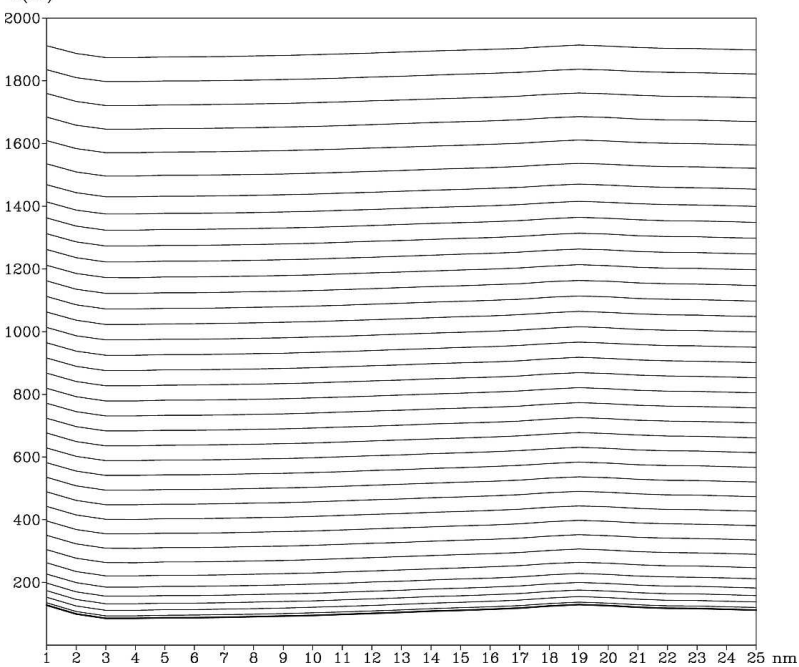

FIG. 1. Grid levels on terrain-following sigma coordinates along the glide path up to a height of $2 \mathrm{~km}$.

ployed. The turbulent energy dissipation rate is extracted from the turbulence kinetic energy (TKE) budget equation solved in the model. Alternatively, we use a simple parameterization discussed in section 4. A multilevel soil vegetation parameterization is employed in our model setup (Smirnova et al. 2000). This combination of the chosen PBL physics and soil model has been validated successfully in earlier applications with model runs of similar resolution (Grell et al. 2000).

For the current application, NOWVIV was adapted to the Frankfurt Airport area. Two nested domains with sizes of $250 \mathrm{~km} \times 250 \mathrm{~km}$ and $90 \mathrm{~km} \times 90 \mathrm{~km}$ centered on Frankfurt Airport with grid distances of 6.3 and $2.1 \mathrm{~km}$, respectively, were used. The model employs 60 vertical levels. The model top is at $200 \mathrm{hPa}$. In the altitude range of interest $(z<1100 \mathrm{~m}$ above ground), the atmosphere is resolved by 26 levels with vertical resolution increasing from 8 to $50 \mathrm{~m}$ (Fig. 1). Initial and boundary data were taken from the mesoscale model named the Local Model (LM) of the German Weather Service (DWD LM; Steppeler et al. 2003) running in data assimilation mode. LM is run at a horizontal resolution of $7 \mathrm{~km}$. These data represent the best possible forcing of NOWVIV since actual observations (radio soundings, satellite data, surface observations, etc.) are used to analyze the state of the atmosphere in the LM. Lateral boundary data are updated every hour using a relaxation scheme. Thereby the outer boundary of the MM5 grid is specified by time-dependent values from the LM, and the adjacent inner MM5 grid points are relaxed toward the boundary values with a constant relaxation that decreases linearly away from the boundary. Detailed topography, land use, and soil type data for the Frankfurt area were employed using a land use database in which data are available at $1-\mathrm{km}$ resolution (Masson et al. 2003). Individual airport buildings are not resolved in this model setup.

From the model runs, profiles of meteorological data were extracted at 25 locations separated by one nautical mile along the glide paths for approaches on the 07 and 25 runways (see Fig. 2). An output frequency of $10 \mathrm{~min}$ was selected. The resulting number of profiles for the 1 -yr database amounts to about $1.3 \times 10^{6}$. The meteorological quantities comprise the three wind components, air density, virtual potential temperature, turbulence kinetic energy, eddy dissipation rate (EDR), and pressure.

\section{Verification against climatological data}

The results of the 1-yr simulation of actual weather around the Frankfurt terminal area are compared with a 30-yr surface wind climatology based on mean wind measurements at Frankfurt Airport from 1967 to 1997. The 30-yr climatology considers winds averaged over 1 $\mathrm{h}$ measured at $10 \mathrm{~m}$ above ground. NOWVIV winds of the first model level at $8 \mathrm{~m}$ above ground are analyzed. Grid point number 14 close to the threshold of runway $25 \mathrm{~L} / \mathrm{R}$ is chosen for the comparison.

The 1-yr simulation covers a period from 1 November 2003 to 31 October 2004. The working hypothesis is that this 1 -yr representation of daily weather is already close to climatological conditions at the airport. Furthermore, if there is a good agreement we can conclude also that forecasted profiles of wind, temperature, and turbulence are close to reality even though we have limited profile observations for verification. The argument here is that the surface winds are the result of the initial forcing and the representation of orography and land use in the model. But more important, the surface winds are very much determined by the quality of the parameterized boundary layer processes. Systematic deficiencies in the boundary layer parameterizations may be reflected in differences between the joint frequency distribution of surface wind speed and direction from NOWVIV output and from the long-term climatology.

The joint frequency distribution of wind speed and direction from the 1-yr NOWVIV run is shown in Fig. 3 (top panel). The comparison with the 30-yr climatology (bottom panel) shows in general good agreement. The Frankfurt wind climatology is characterized by two main wind directions: southwesterly winds with a peak around $200^{\circ}$ and northeasterly winds around $50^{\circ}$. For both main wind directions the corresponding peak in 


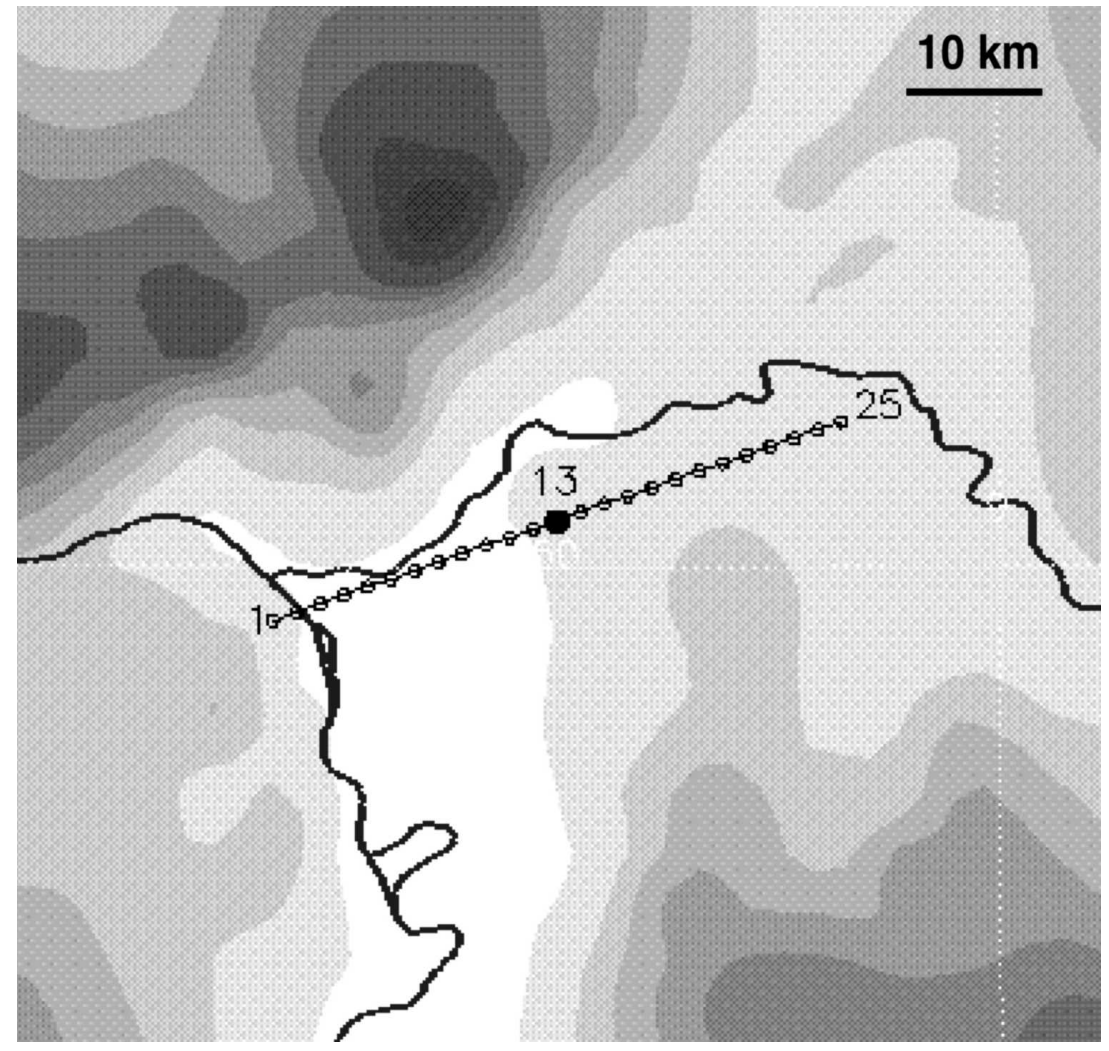

FIG. 2. Locations of 25 meteorological profiles along the glide paths for approaches on the 07 and 25 runways spaced in steps of $1 \mathrm{n} \mathrm{mi}$. The height contours of the orography in Frankfurt are shown in gray shading at 100-m resolution. Gridpoint 13 denotes the location of Frankfurt Airport, located $100 \mathrm{~m}$ above sea level. The map encompasses an area of about $80 \mathrm{~km}^{2}$.

the mean wind speed is between 2 and $4 \mathrm{~m} \mathrm{~s}^{-1}$. The $1-\mathrm{yr}$ NOWVIV run recovers these main features very well. Recall that the main surface wind directions are not only the result of predominant synoptic patterns but also are influenced by the orography in the vicinity of the airport, here in particular the Taunus Mountain ridge.

We notice that there is a shift from easterly winds $\left(50^{\circ}-70^{\circ}\right)$ in the climatology to northeasterly directions $\left(350^{\circ}-40^{\circ}\right)$ in the $1-y r$ NOWVIV data. Altogether, the 1 -yr data more frequently exhibit winds coming from northeasterly directions (sector $350^{\circ}-70^{\circ}, 0-4.5 \mathrm{~m} \mathrm{~s}^{-1}$ ) as compared with the 30-yr climatology. Furthermore, in the 1-yr dataset more frequently (up to $15 \%$ ) winds from $140^{\circ}$ to $220^{\circ}$ are found with wind speeds between 0 and $4 \mathrm{~m} \mathrm{~s}^{-1}$. The occurrence of stronger winds is slightly underrepresented (less than 5\%) as compared with the DWD 30-year climatology.

These differences may be due to the chosen simulation period and the prevailing pressure patterns over central Europe. Part of the underestimation may be related to the finite spatial resolution of the model where the wind is a grid box averaged quantity that is compared with a point measurement. Last, the differences may be attributable to climate variability and trends. For example, in accordance with NOWVIV predictions, a higher frequency of easterly winds is noted by controllers at Frankfurt Airport in recent years with more frequent landings on runway 07 (Frech et al. 2002). A more detailed analysis of the 30 -yr climatology by considering shorter time intervals and more recent data could be used to quantify trends.

\section{Verification against Frankfurt 2004 measurements}

The Frankfurt measurement campaign represents a unique opportunity to assess a subset of the NOWVIV database in more detail by using sonic anemometer and SODAR/RASS measurements. We focus here on a data comparison for 40 days (26 August 2004 until 5 October 2004). The measurements were taken at a site close to the runway threshold of $25 \mathrm{~L} / \mathrm{R}$. This time period includes a whole range of synoptic conditions, 

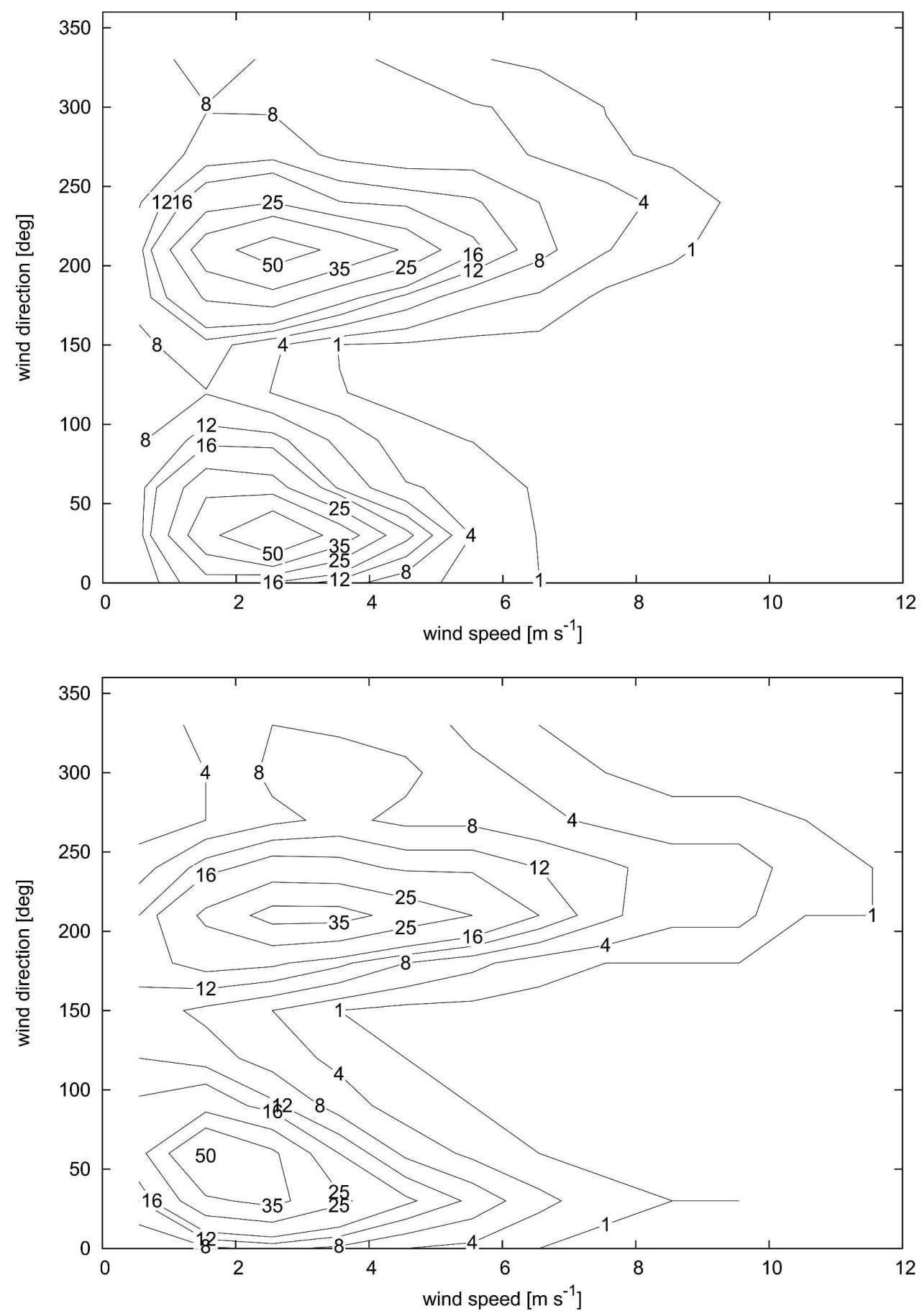

FIG. 3. Joint frequency distribution of wind speed and direction (\%) based on (top) the 1-yr NOWVIV run (November 2003-October 2004) and (bottom) the Frankfurt Airport 30-yr climatology (1967-1997).

from late summer high pressure situations to frontal passages with strong winds.

A METEK, Inc., SODAR DSDPA.90-64 with a 1290-MHz RASS and a USA-1 sonic anemometer at $10-\mathrm{m}$ height were deployed close to the thresholds of runway 25L and 25R (Fig. 4). The SODAR/RASS mea- surements provide 10-min averaged profiles of all 3 wind components, standard deviation of vertical velocity, and virtual temperature. The vertical resolution of the profiles is $20 \mathrm{~m}$, and the first measurement level is $40 \mathrm{~m}$ (which represents an average between 30 and 50 $\mathrm{m})$. The maximum range was set to $300 \mathrm{~m}$. The vertical 


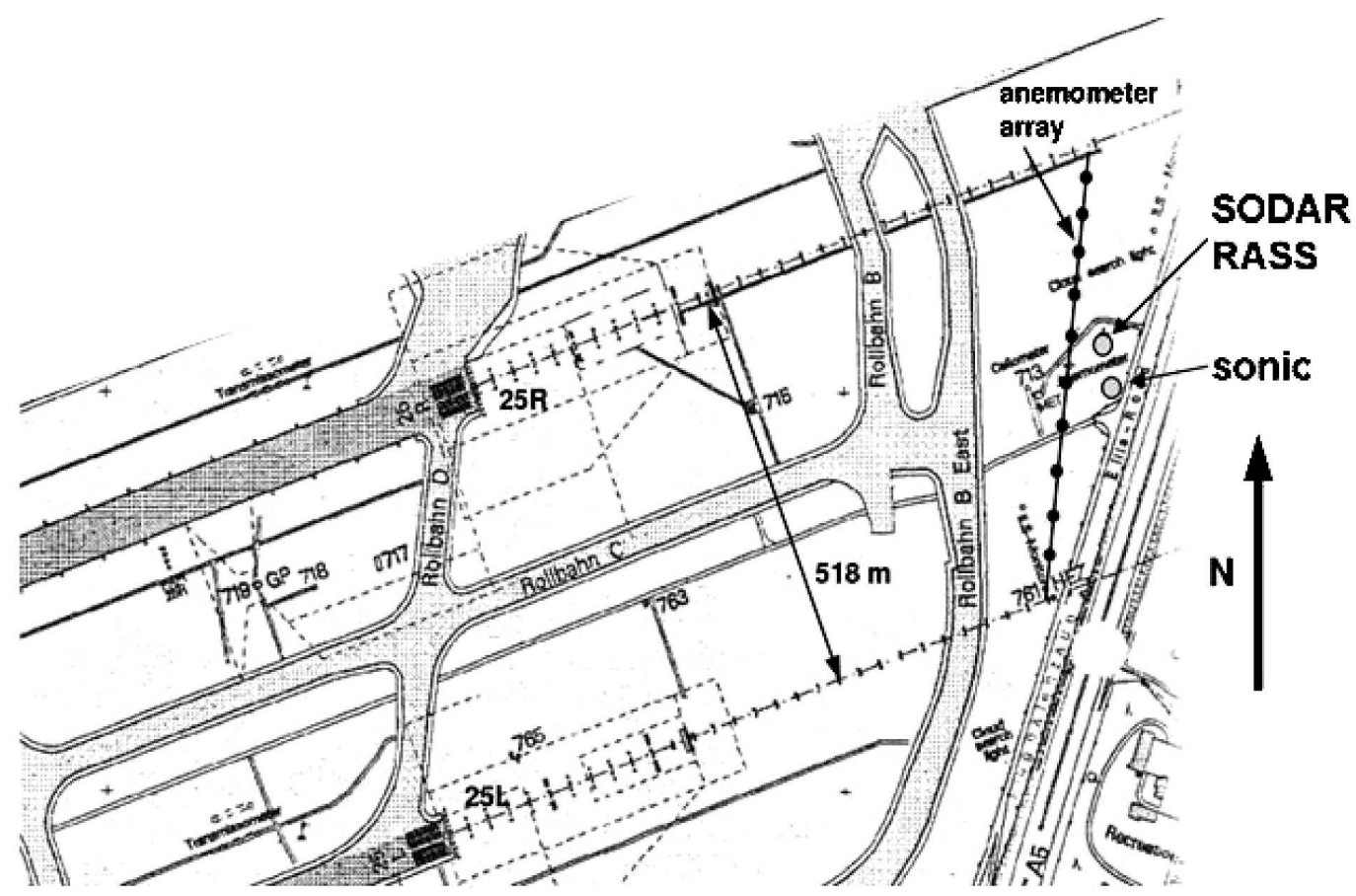

FIG. 4. Sensor locations and runway layout at Frankfurt Airport.

availability of meteorological parameters varies with time depending on environmental (aircraft noise) and meteorological conditions. The accuracy of the wind speed measurement depends on the wind speed magnitude. Up to $5 \mathrm{~m} \mathrm{~s}^{-1}$ the accuracy is within $\pm 0.5 \mathrm{~m} \mathrm{~s}^{-1}$ and from 5 to $35 \mathrm{~m} \mathrm{~s}^{-1}$ within $\pm 10 \%$. For wind direction, the accuracy is $\pm 5^{\circ}$. The standard deviation of vertical wind velocity can be estimated within \pm 0.15 $\mathrm{m} \mathrm{s}^{-1}$. The sonic anemometer provides measurements of all three wind components and temperature at a sampling rate of $17 \mathrm{~Hz}$.

The overall performance of the model in predicting environmental parameters such as wind speed and direction, stratification, shear, and turbulent energy dissipation rate during the aforementioned 40 days is shown in the following. These parameters govern the transport and decay of wake vortices.

Since transport of wake vortices out of the flight corridor is the main hazard reduction mechanism, it is of prime interest to investigate the ability of the model to reproduce the correct frequency of occurrence of wind speed and direction. The height levels considered are 10,100 , and $200 \mathrm{~m}$, a height range where the assessment of wake vortex risk is most important. The NOWVIV forecasts are interpolated to these height levels.

The observed and predicted joint frequency distributions of wind speed and direction for the aforementioned height levels are shown in Figs. 5-7. The sample size for the lowest altitude is on the order of 6000 and decreases to about 2000 samples for the 200-m height level because of variable vertical availability of SODAR measurements. In general we find that the frequency distributions are met well by the model for all height levels. Even for this short period of 40 days we recover the main features of the climatological conditions at Frankfurt Airport with the two main wind directions. Close to the surface we notice an underestimation of the peak velocities of southwesterly winds while the frequency of low winds $\left(<5 \mathrm{~m} \mathrm{~s}^{-1}\right)$ is recovered by the model. Note that part of the measured peak velocities of this wind direction sector may be attributable to the velocity signal of wake vortices, which were not filtered out from of the measurements. Only a slight underestimation of the peak velocity is also found for the easterly direction sector. For these cases we do not expect any bias in the observations because of wake vortices, since only departing aircraft pass the location of the sonic anemometer at a much higher altitude relative to landing aircraft on runways $25 \mathrm{~L} / \mathrm{R}$. Close to the surface, local features such as buildings in the vicinity of the measurement system may in addition cause further deviations between prediction and observation. The effect of local land surface features is diminishing with height and is blended by turbulent diffusion.

The figures also indicate the expected increase of wind speed with height and a turning of the wind vector with height. This in particular can be seen at the 200-m height level where the winds on average are more often 

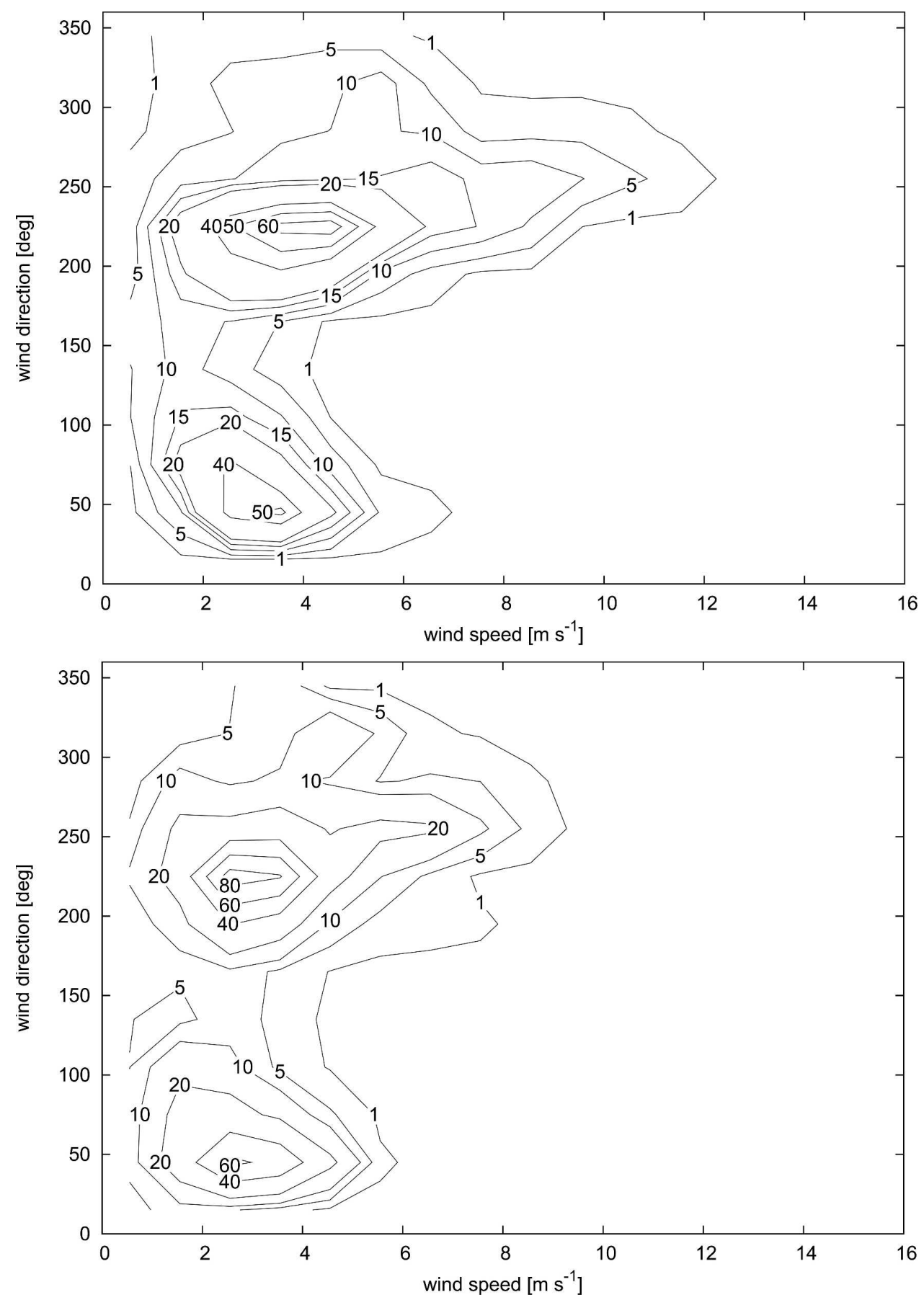

FIG. 5. Joint frequency distribution of (top) observed and (bottom) predicted wind speed and direction at $z=10 \mathrm{~m}(\%)$ during the measurement campaign at Frankfurt Airport. Wind is measured by the sonic anemometer.

westerly as compared with, for example, the 10-m level, where winds have a more southwesterly direction. This clockwise turning of the wind vector with height is the consequence of the balance between vertical turbulent diffusion, horizontal pressure gradient, and Coriolis force. Depending on the wind direction in the free atmosphere, orographic features such as a the Rhine valley can lead to deviations from this simplified balance. Veering or backing of the wind vector due to warm- or cold-air advection may be superposed. 

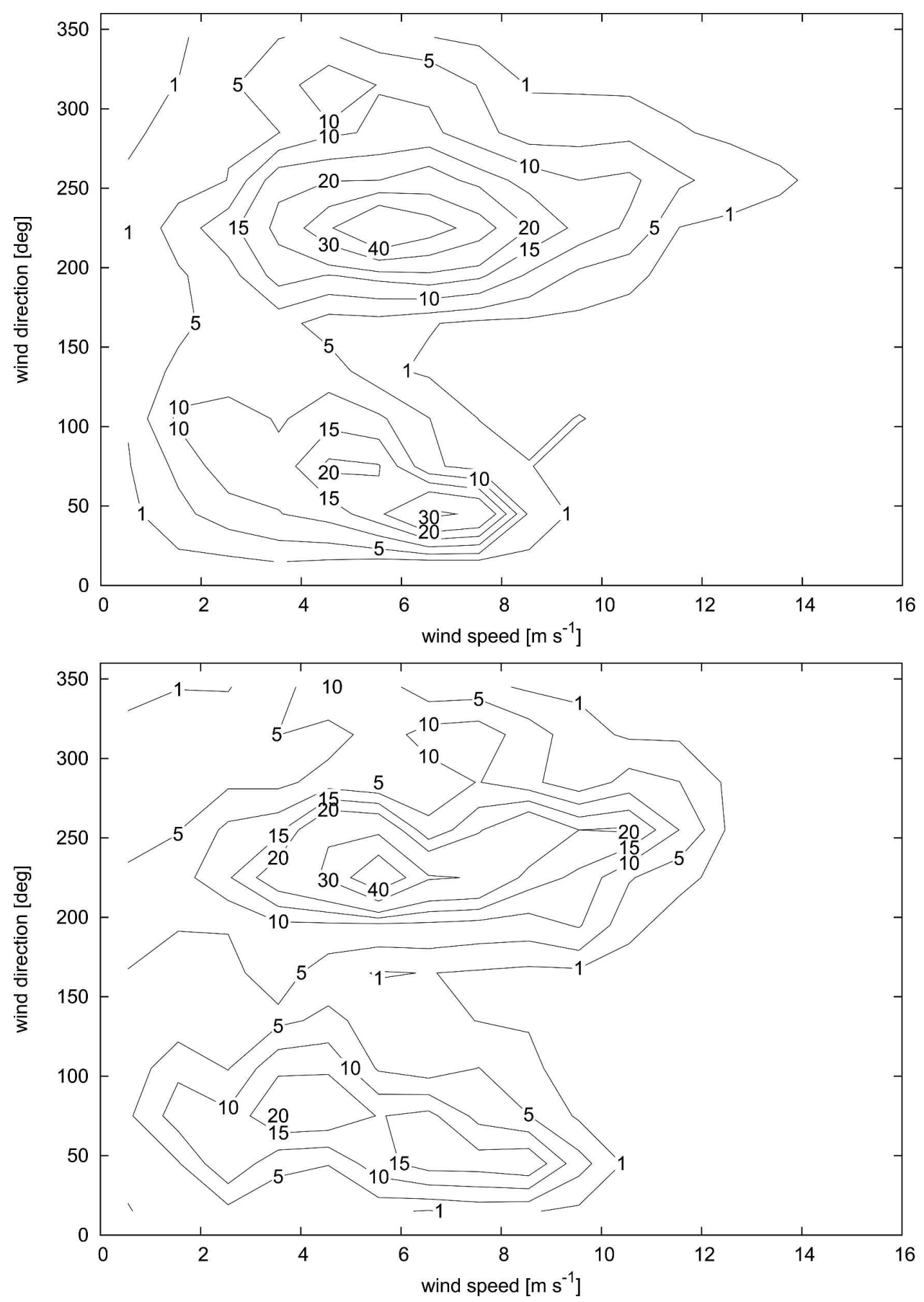

FIG. 6. Joint frequency distribution of (top) observed and (bottom) predicted wind speed and direction at $z=100 \mathrm{~m} \mathrm{( \% )}$. Wind is measured by the SODAR. See also Fig. 5.

For all SODAR height levels, the peak velocity is well reproduced by the model. Wakes are implicitly filtered out from the SODAR measurements during quality control so that we do not expect any wake vortex-related peak velocity bias. Overall, the joint frequency distribution of wind speed and direction, in par- ticular at the 100- and 200-m levels, are reproduced by the model very well.

One key quantity for wake risk assessment is the runway crosswind. It is important that in particular weak crosswind situations are well predicted by the model not only close to the surface but also along the 

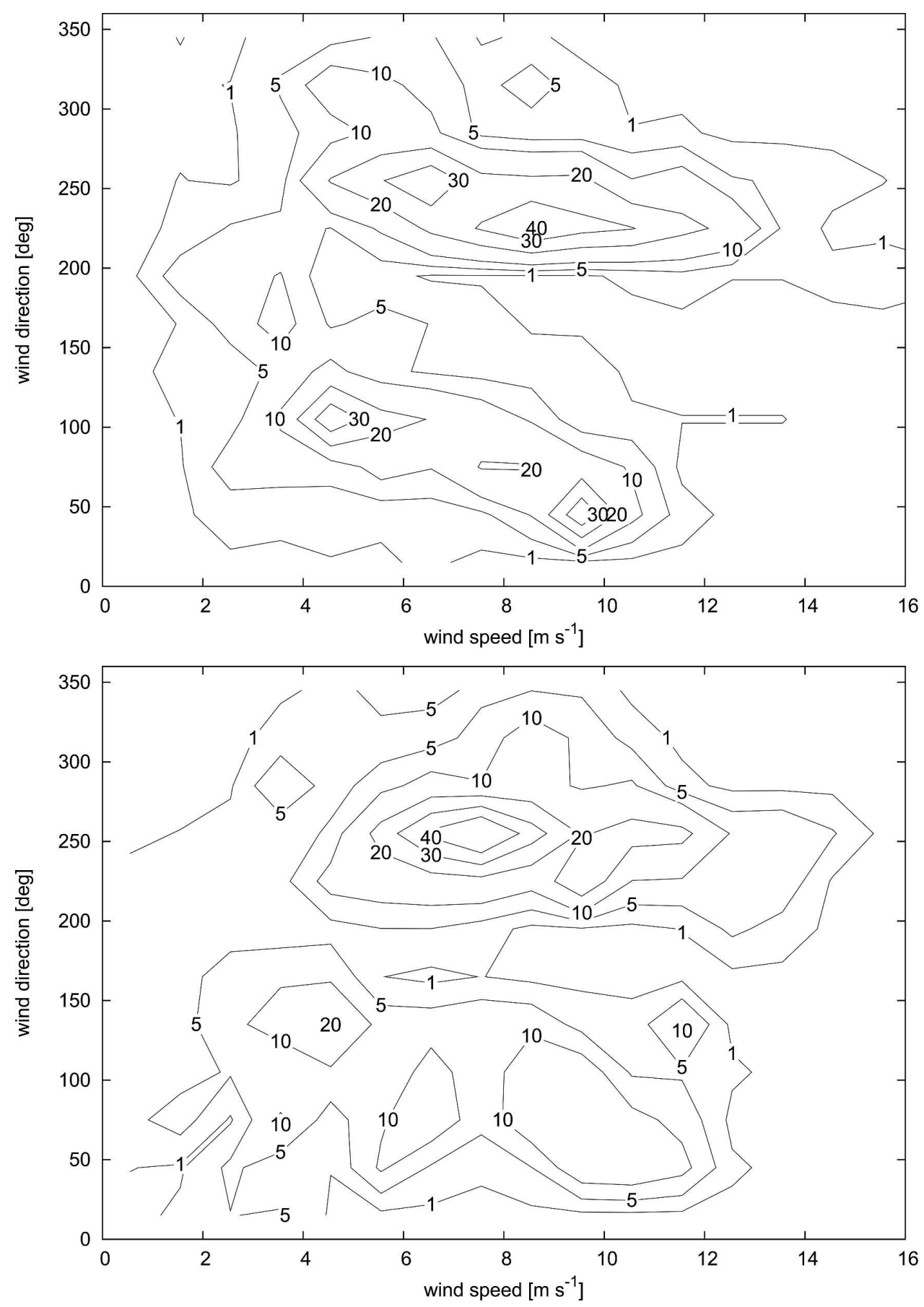

FIG. 7. Joint frequency distribution of (top) observed and (bottom) predicted wind speed and direction at $z=200 \mathrm{~m} \mathrm{( \% )}$. Wind is measured by the SODAR. See also Fig. 5.

glide path, since wake vortices may remain in the flight corridor for relatively long times. Furthermore, for a risk assessment it is important also that the frequency of occurrence of crosswind levels is well represented.

For this purpose, the frequency of occurrence of a runway crosswind bin is given in percent. A positive crosswind is defined as a wind blowing toward a southerly direction $\left(160^{\circ}\right)$. The histogram of the 10 -m wind corresponding to the measurement campaign (Fig. 8, left-hand graph) is skewed toward negative crosswinds for both observation and prediction. This indicates the predominance of winds from south-southwesterly di- 

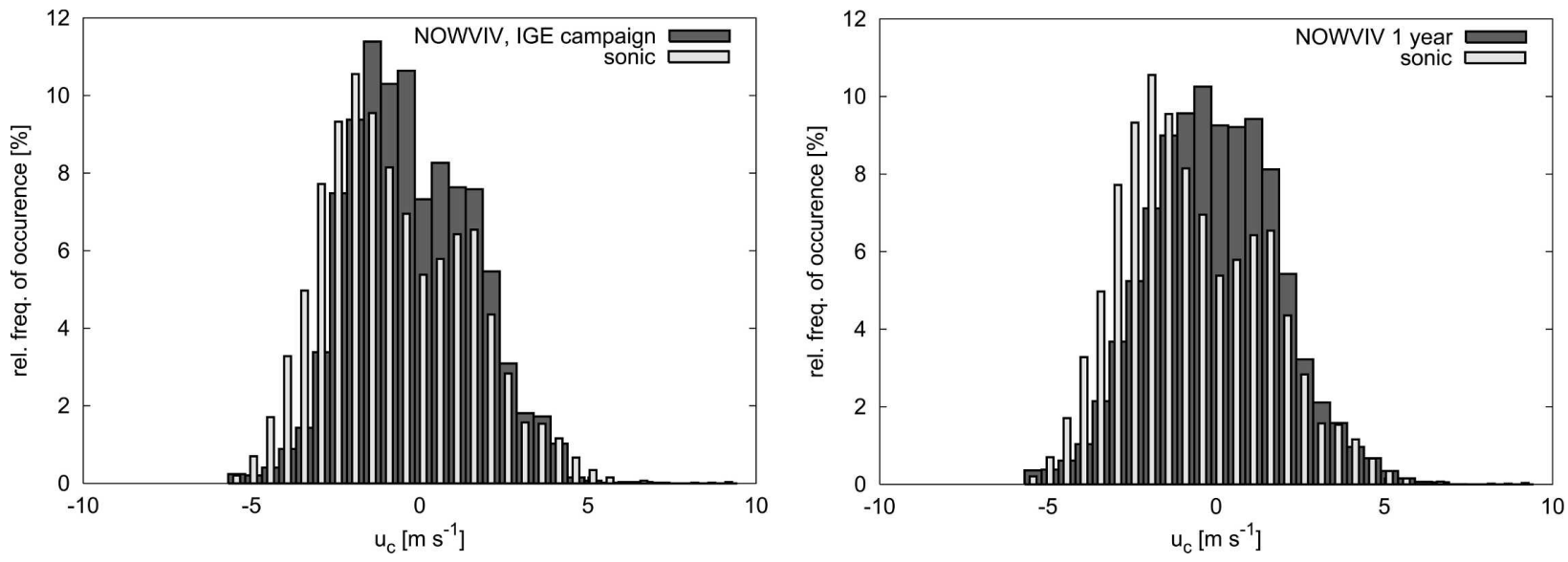

FIG. 8. Histogram of crosswind at $z=10$ : (left) NOWVIV data during the in-ground effect (IGE) campaign and (right) 1-yr NOWVIV database against crosswind measured by ultrasonic anemometer. Sonic data are from the IGE campaign (40 days).

rections $\left(<250^{\circ}\right)$ or from the east $\left(>70^{\circ}\right)$. This histogram shows that crosswinds smaller than $\left|u_{c}\right|<2 \mathrm{~m} \mathrm{~s}^{-1}$ are predicted more frequently than observed, which is also found for the other height levels. The maximum difference between observation and prediction for a given bin is $4 \%$. For comparison, we also show the histogram of the 1-yr database (Fig. 8, right-hand graph). The 1-yr histogram shows roughly a normal distributed crosswind distribution. There is a higher frequency of weak positive crosswinds, which is an indicator for more frequent synoptic situations with westerly $\left(>250^{\circ}\right)$ or northeasterly winds $\left(<70^{\circ}\right)$ in the course of the simulated year.

The histograms for the 100- and 200-m height levels also show good agreement of measurements and predictions with maximum deviations on the order of $4 \%$ (Fig. 9). Again, we find a tendency that weak crosswinds are more frequently predicted than observed, but the overall differences are small.

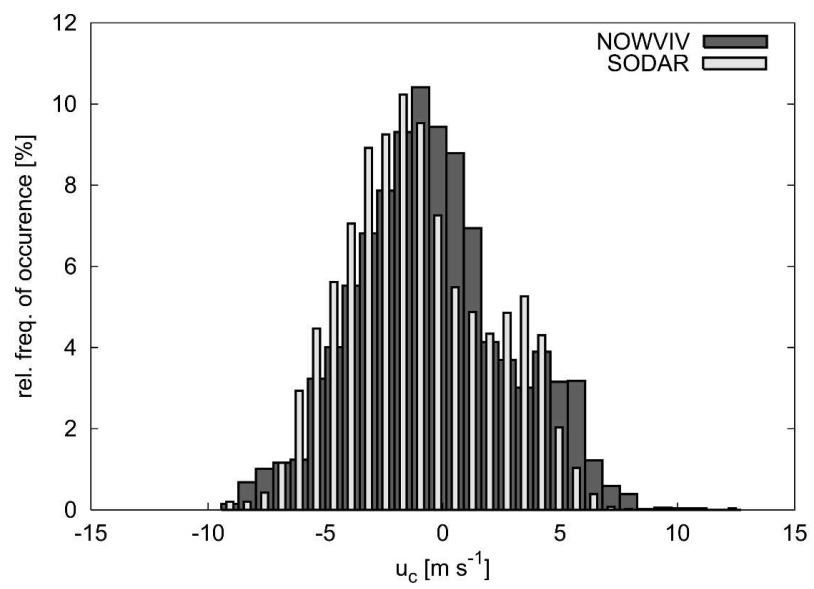

For the measurement campaign, the overall bias and root-mean-square (RMS) error of the model for wind is shown in Fig. 10. The RMS error of wind speed is on the order of $1.5-2 \mathrm{~m} \mathrm{~s}^{-1}$ and slightly increases with height. The mean bias (median of difference between observation and prediction) is close to zero and drops to $-0.5 \mathrm{~m} \mathrm{~s}^{-1}$ at $z=300 \mathrm{~m}$, which indicates no systematic bias in wind speed prediction. The RMS error of wind direction is around $45^{\circ}$ close to the surface and decreases to about $40^{\circ}$ before increasing to $50^{\circ}$ at $z=$ $300 \mathrm{~m}$. The mean bias close to the surface is close to zero and remains almost constant with height. The first and third quartile of the wind direction bias is between $10^{\circ}$ and $20^{\circ}$ and also shows no strong height dependence. The RMS error is dominated by a few outliers, which may relate to situations where the wind speed is weak with no preferred wind direction and to situations with phase errors in the evolution of predicted synoptic fronts. This is substantiated using the joint frequency

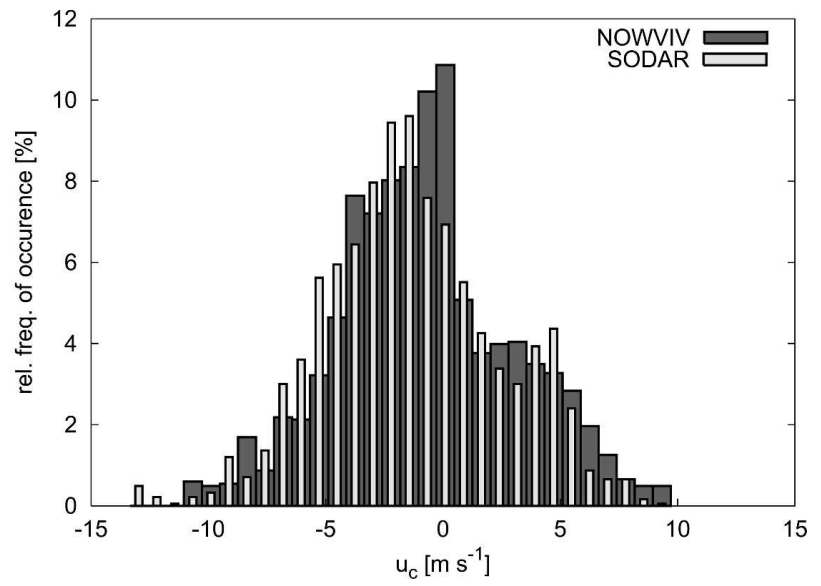

FIG. 9. Histogram of crosswind at $z=$ (left) 100 and (right) $200 \mathrm{~m}$ during the IGE campaign (40 days). 

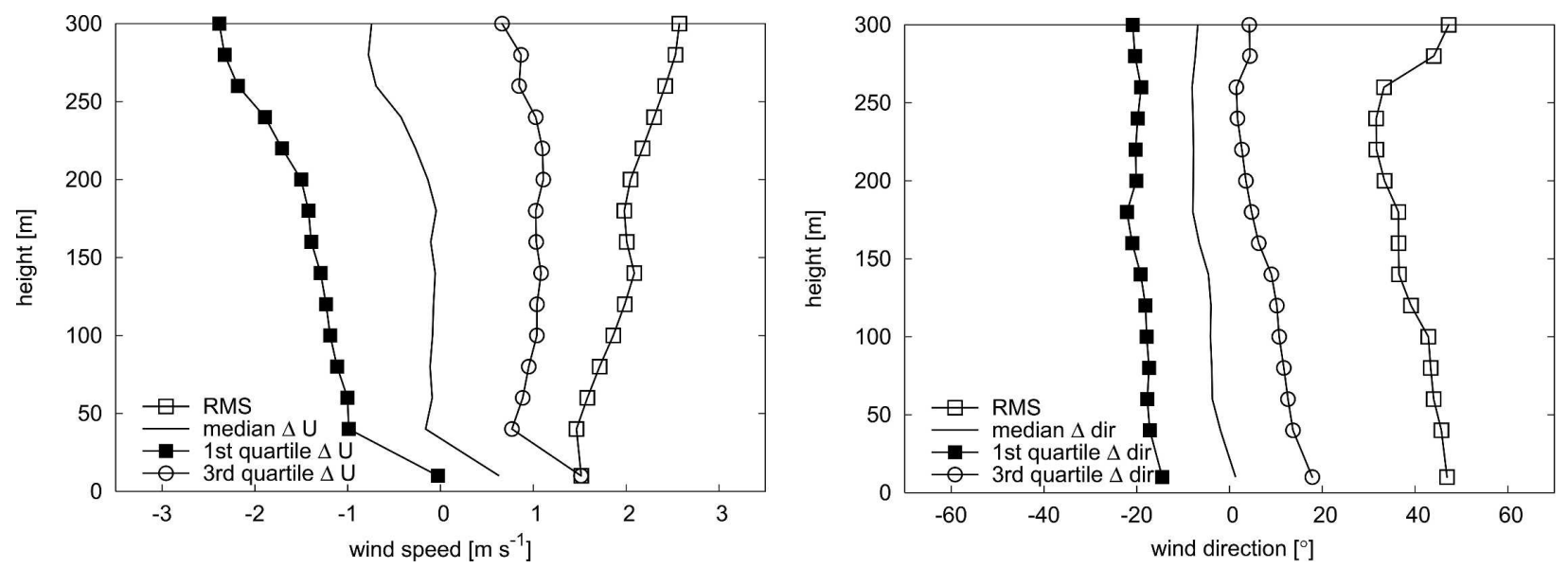

FIG. 10. RMSE and mean bias of wind speed and direction based on 40 days.

distribution of model-predicted wind speed and wind direction bias (Fig. 11). This plot shows that the wind direction error is largest for weak wind speeds and decreases for stronger winds. The percentage of large wind direction errors is small, which supports the previous interpretation that the RMS error is dominated by a few outliers. Another view on the same issue can be obtained from the joint frequency distribution of the biases of wind speed and direction (Fig. 12). Small er- rors in wind speed are more frequently related to large errors in wind direction. Most of those cases appear to be weak wind situations that can be inferred from the spread of the direction bias seen in Figs. 11 and 12. Relative to, for instance, the results shown in Zhong et al. (2005), both the mean bias of wind speed and direction indicate a good forecast performance for those 40 days.

We now investigate the performance of the model to

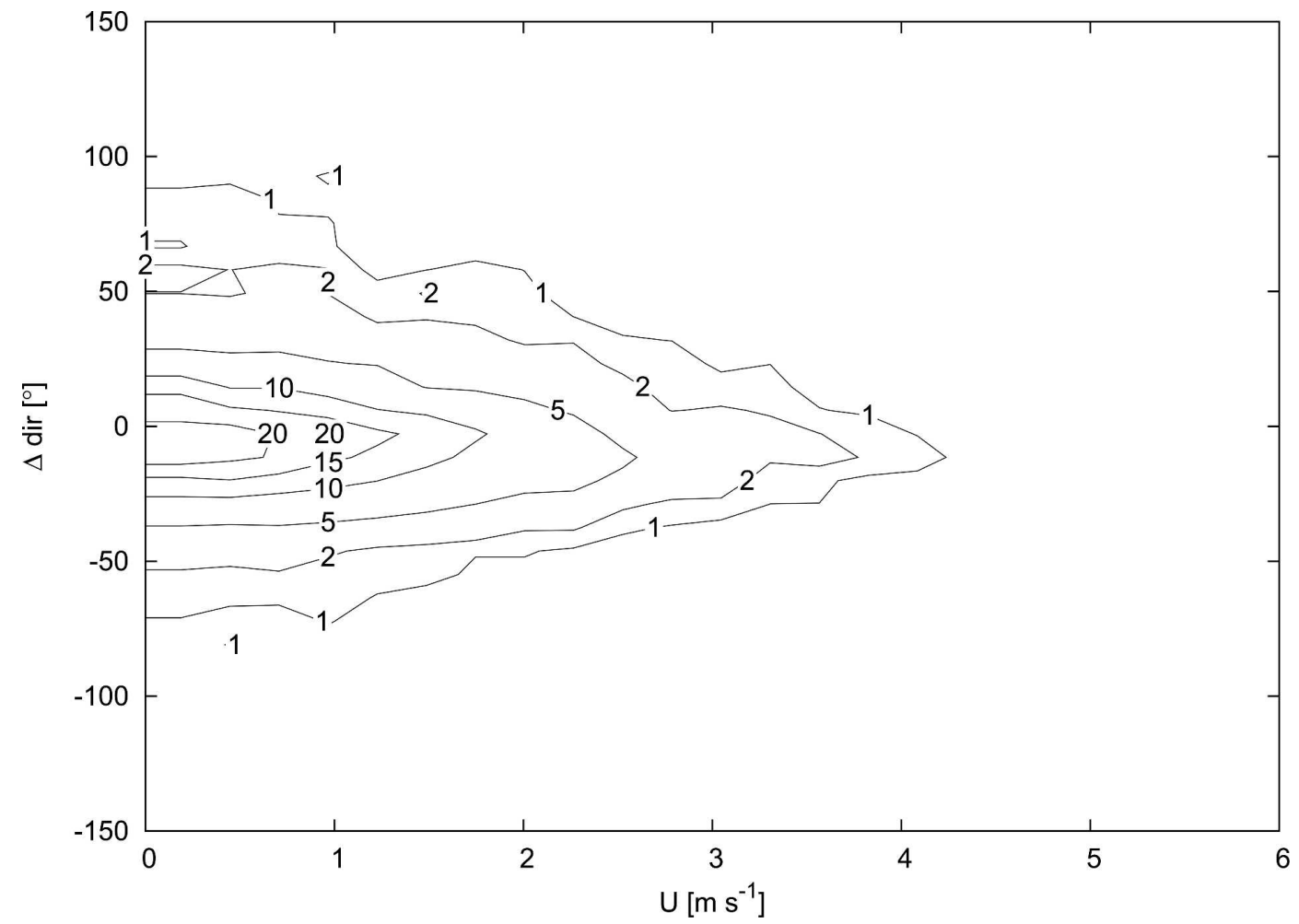

FIG. 11. Joint frequency distribution of model-predicted wind speed and wind direction bias (\%). 


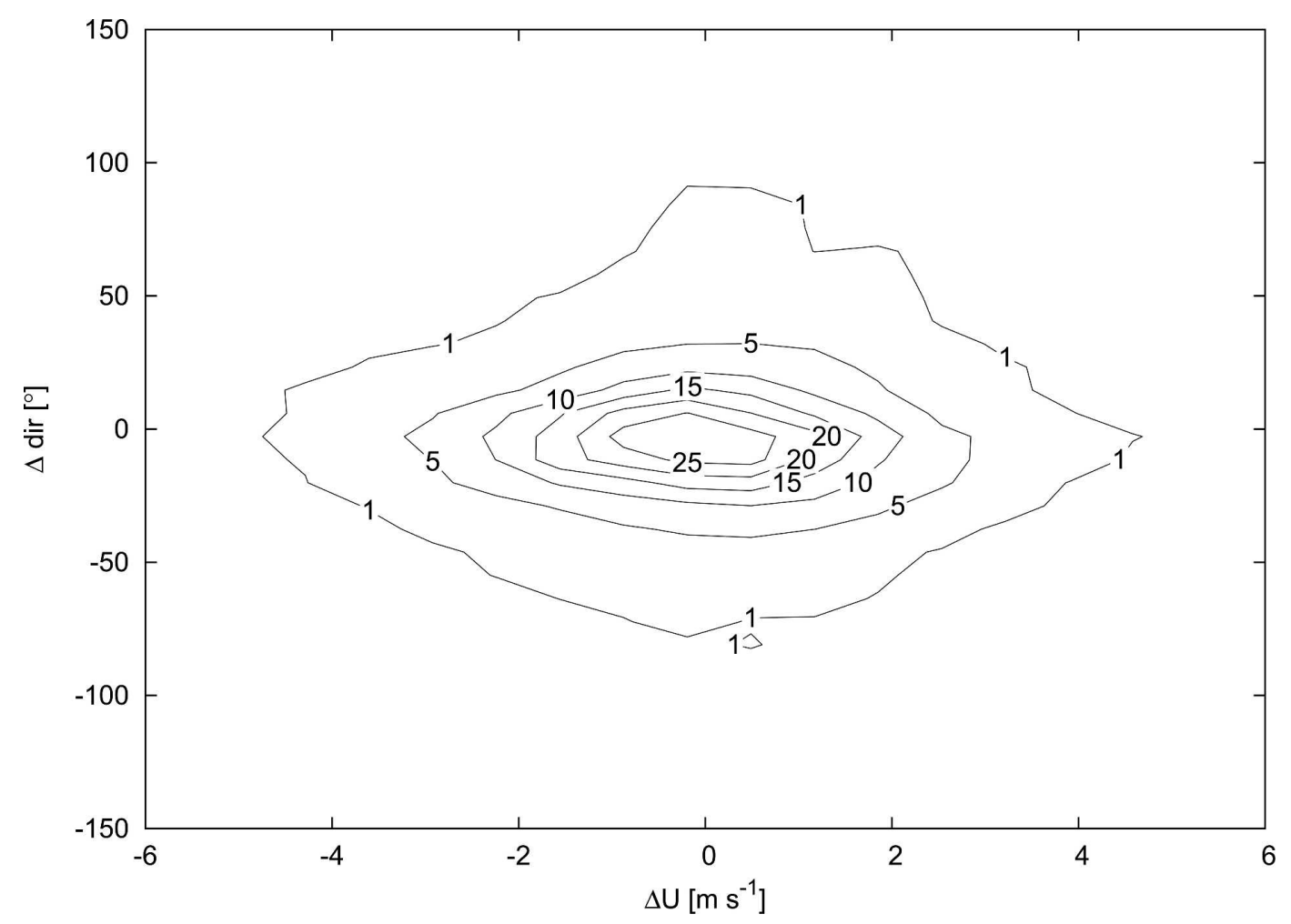

FIG. 12. Joint frequency distribution of wind speed bias vs wind direction bias (\%).

predict stably stratified situations. The Brunt-Väisälä frequency is approximated as

$$
\begin{aligned}
N^{2}(z) & =\frac{g}{\theta_{0 v}} \frac{\partial \theta_{v}}{\partial z} \\
& \approx \frac{g}{\theta_{0 v}} \frac{\theta_{v}\left(z_{i}\right)-\theta_{v}\left(z_{i-1}\right)}{z_{i}-z_{i-1}},
\end{aligned}
$$

with the gravitational constant $g$, the virtual potential temperature $\theta_{v}$, and height level $z$. For $\theta_{0 v}$ we use the virtual potential temperature at height $z_{i}$. For NOWVIV, we first evaluate $N^{2}(z)$ from the predicted profiles before we perform the interpolation of $N^{2}$ to the SODAR/RASS height levels.

The histogram for all $N^{2}$ values up to $200 \mathrm{~m}$ is shown in Fig. 13. We notice the high percentage $(28 \%)$ of near-neutral conditions in the NOWVIV forecast. This is only observed in $13 \%$ of the SODAR/RASS profiles. For the first bin in the stable regime $\left(N^{2}>0 \mathrm{~s}^{-2}\right)$, the difference is smaller and reversed (22\% observed versus $17 \%$ predicted). We will focus our analysis on stable stratified where decelerated wake descent may be expected. The analysis of Holzäpfel et al. (2001) showed that the wake vortex may stall in stable situations where the normalized Brunt-Väisälä frequency has a magnitude of $N^{*} \approx 1$, where $N^{*} \equiv N t_{0}$. Accelerated wake vortex decay is found for $N^{*} \approx 0.5$. Here, $t_{0}$ denotes the characteristic time scale of a wake vortex pair to descend one vortex spacing as a result of mutual velocity induction. For a heavy aircraft $t_{0}$ is on the order of $30 \mathrm{~s}$. The normalized stratification magnitude of $N^{*}=0.5$ is underestimated by the forecast $(23 \%$ observed versus $12 \%$ predicted). A value of $N^{*}=1$ is observed and predicted about $2 \%$ of the time. Overall, the histogram indicates an underestimation of weak to moderate stable stratification by the model relative to observations. In particular, for weak stable situations a 10-min average of potential temperature from SODAR data may be not sufficient to estimate the mean stratification. The observations show that the boundary layer may be turbulent under weak to moderate stable stratification (not shown). Under such circumstances, the model tends to produce well-mixed conditions in the temperature. Strong stably stratified situations, however, occur more frequently in the prediction, even though the absolute numbers and the differences between observation and prediction are small.

We now analyze predictions of shear where we define the absolute shear rate (Sh) at height $z$ as

$$
\operatorname{Sh}(z)=\left\{\left[\frac{u\left(z_{i}\right)-u\left(z_{i-1}\right)}{z_{i}-z_{i-1}}\right]^{2}+\left[\frac{v\left(z_{i}\right)-v\left(z_{i-1}\right)}{z_{i}-z_{i-1}}\right]^{2}\right\}^{1 / 2} .
$$




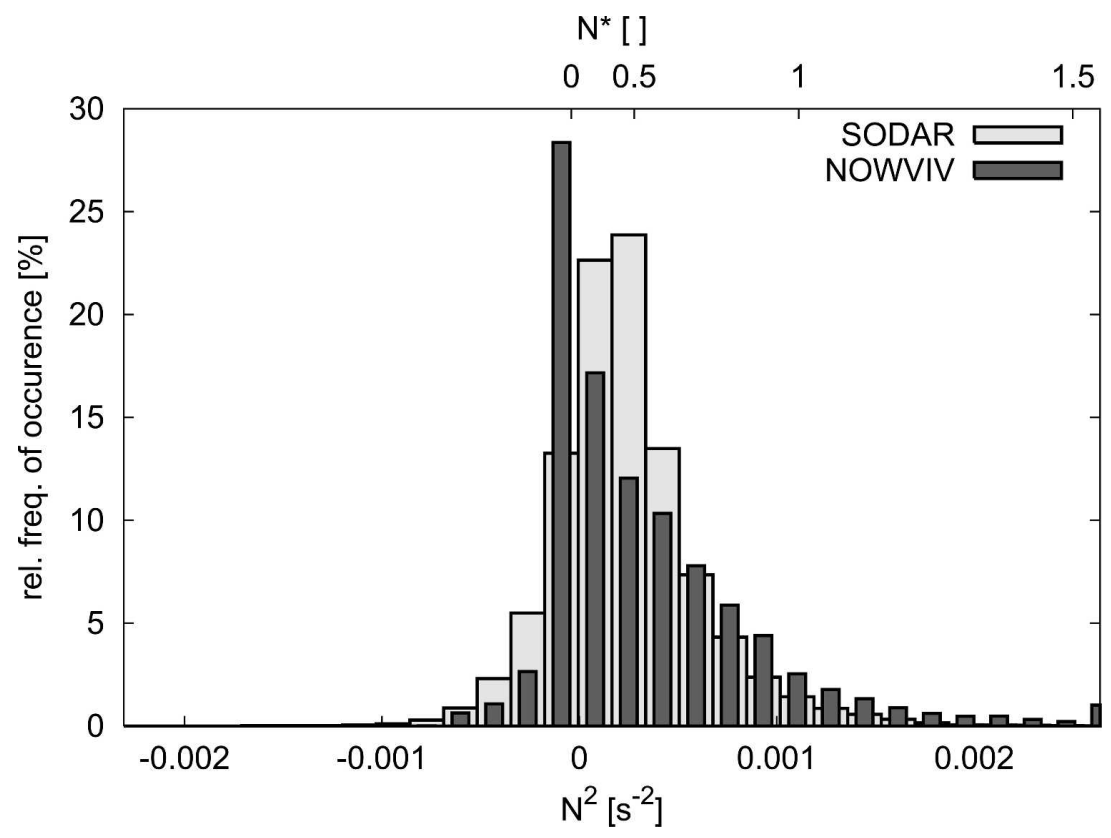

FIG. 13. Histogram of $N^{2}$, all values between 40 and $200 \mathrm{~m}$. In addition, the corresponding normalized Brunt-Väisälä frequency $N^{*}$ for $N^{2}>0$ is shown, where a characteristic time scale $t_{0}=30 \mathrm{~s}$ is assumed.

We first compute shear for each height level from the SODAR and NOWVIV wind profiles. The NOWVIV shear values are then linearly interpolated to the SODAR height levels simply by using the two nearest NOWVIV neighbors relative to the height level $z$. The histogram is interpreted considering the shear parameterization of the wake vortex transport and decay model P2P (Holzäpfel 2005). This model employs a critical normalized shear level Sh* $\equiv \mathrm{Sh} \times t_{0}=1$ for which a stall or rebound of wake vortices (WV) has been observed. Assuming a characteristic time scale of $t_{0}=30 \mathrm{~s}$, this corresponds to a critical shear threshold of $\mathrm{Sh}=0.033 \mathrm{~s}^{-1}$.

We consider the histogram for all shear values between 40 and $200 \mathrm{~m}$ (Fig. 14). In general the agreement between model and observation is good. Larger differences are found in particular for shear levels $\mathrm{Sh}<$ $0.04 \mathrm{~s}^{-1}$ where the largest deviation is found for the smallest shear values ( $9 \%$ points). The model tends to more well-mixed conditions relative to the observations. Similar to situations of weak stable stratification, we note that 10-min averages of observed wind speed may cause uncertainties in the estimation of weak shear levels. The frequency of occurrence of shear levels above the critical shear threshold is very well reproduced by the model. Differences are smaller than $1 \%$ points. Conditions that in principle favor vortex stall and rebound occur $40 \%$ of the time. Whether a vortex actually stalls or rebounds depends on the actual tur- bulence level. Turbulence reduces the lifetime of a wake vortex such that shear effects will not become effective.

Another important variable for predicting wake vortex decay is the turbulent energy dissipation rate $\varepsilon$, which is a good predictor of wake vortex demise in a turbulent atmosphere (Sarpkaya 2000; Holzäpfel 2003). The correlation of demise time and turbulent kinetic energy is weak because large eddies, which contribute most to TKE, do not represent the optimum length scale to trigger a perturbation to the wake vortex symmetry with the subsequent onset of rapid decay due to instability mechanisms. In addition, for operational applications, the reliability of TKE estimates is very much dependent on the choice of the averaging interval in time and space in order to capture all relevant turbulent length scales. Dissipation rate estimates from measurements that employ spectral methods to compute the dissipation rate in the inertial subrange of a power spectrum are more robust in this context (e.g., Piper 2001).

In the model, we have chosen a second-order level-3 turbulence closure scheme. From the budget equation of turbulent kinetic energy we evaluate the dissipation term. We recognize that possible errors and simplifications of the closure scheme may cause a bias of modelcomputed dissipation rates. During the Frankfurt measurement campaign, 413 profiles of lidar-derived eddy dissipation rates with a vertical resolution of $20 \mathrm{~m}$ are available in the altitude range between 20 and $300 \mathrm{~m}$ 


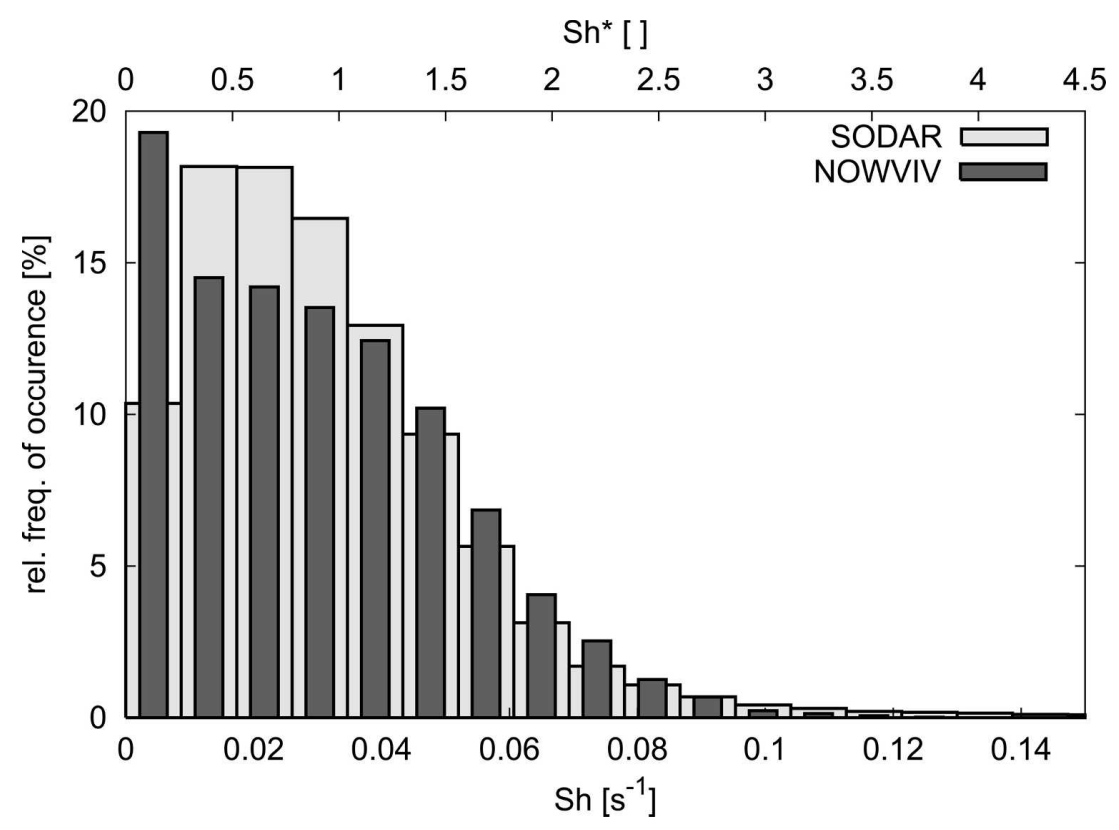

FIG. 14. Histogram of all shear data between $z=40$ and $z=200 \mathrm{~m}$. In addition, the corresponding normalized shear rate Sh* is shown, where a characteristic time scale $t_{0}=$ $30 \mathrm{~s}$ is assumed.

(Smalikho et al. 2005). Lidar EDR data at lower altitudes are not available from this measurement period. The profiles represent 5-min averages. A one-to-one comparison of the 20-m lidar EDR data and reference EDR data derived from sonic data at $10 \mathrm{~m}$ (Frech 2006) shows lower lidar EDR values for $\varepsilon>0.001 \mathrm{~m}^{2} \mathrm{~s}^{-3}$ (Fig. 15). At least in parts, this is consistent with the expected decrease of EDR with height in the boundary layer. For smaller EDR values, that lidar agrees well with EDR estimated from the sonic anemometer. Keeping those uncertainties in mind, we now compare model-predicted EDR profiles with those evaluated by lidar.

For the anaylsis we use direct model output (DMO) and EDR derived as a function of model-predicted TKE according to the empirical formula EDR = $\mathrm{TKE}^{1.5} / 311 \mathrm{~m}$, first proposed by Donaldson and Bilanin (1975). In this simple formula the height-dependent integral length scale is set constant to $311 \mathrm{~m}$ in order to compensate for the overestimate of EDR at low altitudes. The latter relation has been employed in several wake vortex measurement and prediction campaigns (Holzäpfel and Robins 2004).

We first evaluate the RMS error and the distribution of EDR deviations. We consider the mean bias (median) and the first and third quartile of the EDR deviations, where the deviation is defined as $\triangle \mathrm{EDR}=$ $\mathrm{EDR}_{\text {observation }}-\mathrm{EDR}_{\text {prediction. }}$. For an overall view of the error profiles we show Fig. 16. Clearly the DMO data show large deviations very close to the surface that become quickly smaller with height. EDR derived from TKE agrees much better with observations close to the surface. Above $100 \mathrm{~m}$, the DMO EDR performs better in comparison with the derived EDR. The mean bias of the DMO is here on the order of $10^{-4} \mathrm{~m}^{2} \mathrm{~s}^{-3}$, whereas the mean bias of the TKE-derived EDR is slightly larger. But more importantly the overall deviations of EDR derived from TKE are skewed to more positive

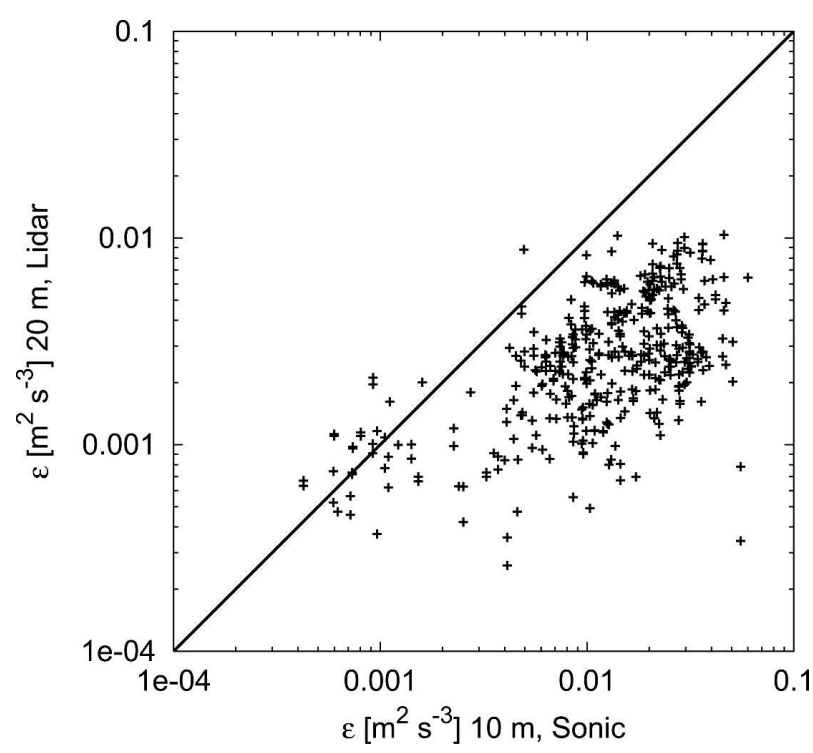

FIG. 15. Scatterplot of EDR derived from sonic anemometer measurements $(z=10 \mathrm{~m})$ and lidar measurements $(z=20 \mathrm{~m})$. 

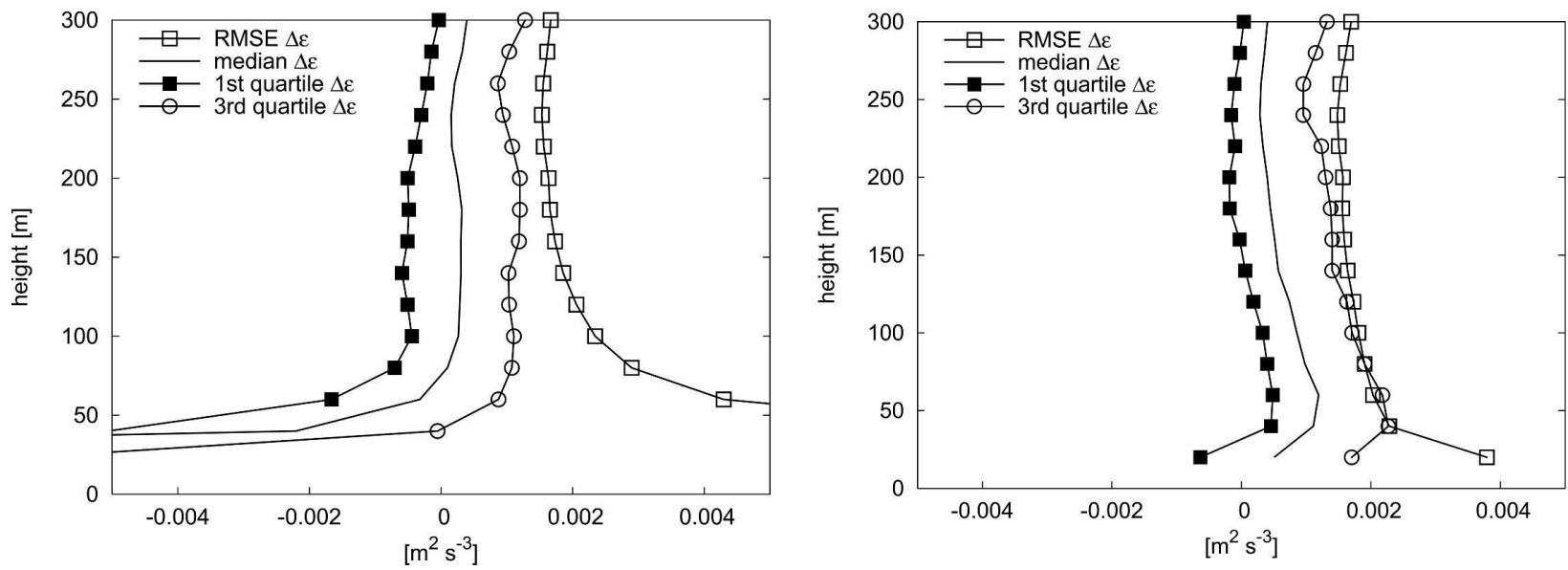

FIG. 16. RMS error of EDR, mean bias, first and third quartile of the EDR deviation based on 413 lidar measured EDR profiles: (left) EDR based on direct model output and (right) EDR derived from TKE.

values indicating the tendency of an underestimation of EDR. Based on this result it can be suggested to use the TKE-derived EDR below $100 \mathrm{~m}$ and the DMO EDR above $100 \mathrm{~m}$. The magnitudes of the RMS errors relative to the curves for the first and third quartiles indicate that the deviations are dominated by a few outliers. Large deviations are related to situations where predicted EDR is very small or zero in cases where the boundary layer is very shallow.

To sum up this chapter on the NOWVIV weather data analysis, we can conclude that the 1-yr database contains weather that is representative of the conditions at Frankfurt Airport. The verification of the model results using measurements taken at the airport suggests that we can also expect realistic weather conditions along the glide path.

\section{Spatial variability of the meteorological fields}

In the previous section we have analyzed model prediction quality. Now, the 1-yr database is used to assess operational approach scenarios that require certain meteorological conditions. So far, the prediction of the lateral transport of wake vortices out of the flight corridor has been the main focus in the development of wake avoidance systems. To realize a reduction of aircraft separation, the crosswind must not change sign along and just below the glide path. Naturally, there are limited observational data to assess the crosswind variability along the glide path. Some insight on spatial and temporal variability of meteorological conditions along the glide path can be investigated using our 1-yr database. Another aspect is the choice of an optimal sensor set up to obtain the best meteorological data coverage for wake vortex prediction. Here we analyze the dataset assuming that only a single wind profiler is available, as is the case at Frankfurt Airport. We investigate to what extent it is possible to map the crosswind variability of a single vertical wind profile on the glide path conditions. Crosswind variability along the glide path may be expected because of model-resolved mesoscale fluctuations and circulations, orography, and land use variability.

We will first investigate the fraction of time where we find a crosswind of $\left|u_{c}\right|>2 \mathrm{~m} \mathrm{~s}^{-1}$ all along the glide path at one instant of time (Frech and Zinner 2004). As an example we compute these statistics for landings on runway 25 by analyzing the meteorological conditions of glide path profile numbers 14-25. In addition it is required that the tailwind at the threshold is smaller than $5 \mathrm{kt}\left(1 \mathrm{kt} \approx 0.5 \mathrm{~m} \mathrm{~s}^{-1}\right)$. For this analysis we adjust the threshold by taking into account the surface wind speed bias of $0.6 \mathrm{~m} \mathrm{~s}^{-1}$ (see Fig. 10). Tailwinds larger than $5 \mathrm{kt}$ typically require a change in landing direction. For each predicted profile we first estimate the glide path height and evaluate the meteorological conditions in a height interval of $200 \mathrm{~m}$ below the glide path (assuming a glide slope angle of $3^{\circ}$ with a glide path intercept at $z=1200 \mathrm{~m}$ and a typical WV descent height of $200 \mathrm{~m}$ ). Then we compute the overall statistics for all glide path profiles and determine the number of situations for which the selected crosswind criterion holds. The results for the 1-yr dataset are summarized in Table 1. Seventy-nine percent of the time the tailwind situation would allow landings on runway $25 \mathrm{~L} / \mathrm{R}$. A crosswind larger than $2 \mathrm{~m} \mathrm{~s}^{-1}$ is found in $30 \%$ of all profiles of the grid point near the runway threshold. If we require that this has to be satisfied along the glide path, the crosswind criterion holds in only $12 \%$ of the cases. If we evaluate only profiles at grid point 12 , we 
TABLE 1. Relative frequency of crosswind conditions $>2 \mathrm{~m} \mathrm{~s}^{-1}$ close to the surface and along the glide path (see text) and for profiles at grid point 12 (GP 12).

\begin{tabular}{ll}
\hline \hline Tailwind $<5 \mathrm{kt}$ & $79 \%$ \\
$\left|u_{c}\right|>2 \mathrm{~m} \mathrm{~s}^{-1}$ at threshold & $30 \%$ \\
$\left|u_{c}\right|>2 \mathrm{~m} \mathrm{~s}^{-1}$ glide path & $12 \%$ \\
$\left|u_{c}\right|>2 \mathrm{~m} \mathrm{~s}^{-1}$ GP 12 & $12 \%$ \\
\hline
\end{tabular}

also find that $12 \%$ of the profiles satisfy the criterion. Grid point 12 is approximately representative of the position of the existing DFS wind profiler. This result indicates that the crosswind variability along the glide path due to wind speed and direction changes with height is substantial. Furthermore, it appears that one wind profiler may be sufficient to capture most of the overall wind variability along the glide path in a climatological sense. Note that we were not analyzing the height level of crosswind changes since a crosswind threshold has to be satisfied all along the glide path. That means that the crosswind change seen along the glide path has to be captured by the wind profiler but not precisely at the altitude at which it occurs. The result of this diagnostic study of course is limited because we do not consider the uncertainties of the predictions. Nevertheless, such an analysis can provide some insight into potential benefits and limitations of new WV prediction systems.

An illustrative example of typical crosswind variability is shown in Fig. 17 where the crosswind and the corresponding horizontal wind vector are shown at 1600 UTC 20 September 2004. For most of the glide path to runway 25 (grid points 14-25), crosswinds stronger than $2 \mathrm{~m} \mathrm{~s}^{-1}$ are found, while smaller magnitudes are found close to the surface. Close to the surface winds are weak. Even though a good part of the glide path has favorable crosswind conditions, the situation close to the surface does not allow for changes in aircraft separations for a WV prediction system solely relying on lateral wake transport.

Now we investigate to what extent the wind profile measured by a wind profiler is representative of the conditions along the glide path at a given time. For this purpose we consider the crosswinds between the first model level and the intercept height at around $1200 \mathrm{~m}$ and compute the minimum crosswind found in this height range for each profile. The same is computed along the glide path where we evaluate the minimum crosswind in a height interval between the glide path elevation at a given grid point and $200 \mathrm{~m}$ below the respective elevation. The overall minimum crosswind is then computed for the whole glide path. The difference between the minimum crosswind along the glide path and grid point 12 is then computed and analyzed. This analysis is carried out separately for the glide paths for landings on runway 25 and 07 in order to identify any systematic differences of meteorological conditions due to, for example, orography. The results are summarized in Table 2.

The results indicate only small differences for the two glide paths. The median difference is $-0.2 \mathrm{~m} \mathrm{~s}^{-1}$ for 25 $\mathrm{L} / \mathrm{R}$ and there is no difference for $07 \mathrm{~L} / \mathrm{R}$. The numbers in Table 2 appear to indicate that there is a weak ten-

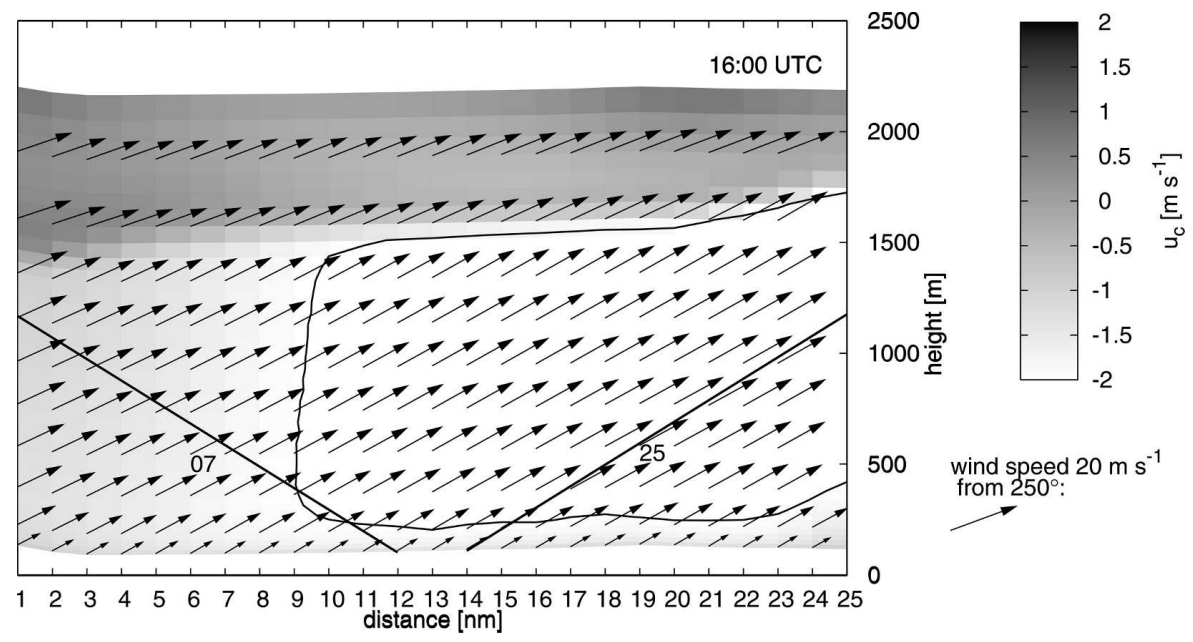

FIG. 17. Wind variability along a glide path cross section at 1600 UTC 20 Sep 2004. Shown is the horizontal wind vector as function of height and the corresponding crosswind along the cross section. Glide paths to landing thresholds $25 \mathrm{~L} / \mathrm{R}$ (grid points $14-25$ ) and $07 \mathrm{~L} / \mathrm{R}$ (grid points 1-12) are denoted by solid lines. The $-2 \mathrm{~m} \mathrm{~s}^{-1}$ crosswind contour line is shown to identify areas with $u_{c}<-2 \mathrm{~m} \mathrm{~s}^{-1}$. 
TABLE 2. Differences between minimum crosswinds along the glide path and grid point $12\left(\mathrm{~m} \mathrm{~s}^{-1}\right)$.

\begin{tabular}{lcc}
\hline \hline & $25 \mathrm{~L} / \mathrm{R}$ & $07 \mathrm{~L} / \mathrm{R}$ \\
\hline Min & -8.7 & -7.3 \\
1st quartile & -0.6 & -0.3 \\
Median & -0.2 & 0.0 \\
3rd quartile & 0.2 & 0.2 \\
Max & 7.0 & 7.3 \\
\hline
\end{tabular}

dency toward more homogeneous crosswind conditions along glide path 07 , which may be attributed to different orographic features. The few large differences may be attributed to frontal systems or thunderstorms that naturally cause a strong spatial heterogeneity of the wind field at the mesoscale. The frequency of occurrence of those events is, however, small. A better view can be gained if we consider the joint frequency distribution of minimum crosswind along the glide path versus the minimum crosswind at grid point 12 (Fig. 18). The figure gives an impression on the range of crosswind sign and magnitude extending from $-15 \mathrm{~m} \mathrm{~s}^{-1}$ up to $6 \mathrm{~m} \mathrm{~s}^{-1}$.
The spatial variability of crosswind along the glide path may be predicted statistically for a single wind profiler. In this approach, the spatial variability is accounted for by the definition of appropriate safety allowances that have to be added to the minimum (maximum) synthetic profiler crosswind. For this purpose we initially bin the full-year synthetic profiler data in 40 intervals of minimum crosswind determined in a height range from 8 to $1200 \mathrm{~m}$. We then subtract the minimum profiler crosswind from the minimum crosswind along the glide path for each bin, whereas the differences represent the spatial variability of crosswind with respect to the profiler. The same is done for the maximum crosswind. Then a cumulative distribution is determined from which the 5th and 95th percentiles are computed. These percentiles define the safety allowances that have to be added to the profiler crosswind in order to guarantee a certain crosswind threshold along the glide path. The results are shown in Fig. 19. Applying a linear fit to the 5th and 95th percentile curves, the crosswind variability can be expressed as $u_{c, \min , 5}=1.00 u_{c, \text { min,profiler }}-1.77 \mathrm{~m} \mathrm{~s}^{-1}$ and $u_{c, \min , 95}=$ $0.95 u_{c, \text { min,profiler }}+0.71 \mathrm{~m} \mathrm{~s}^{-1}$, respectively. Similarly,

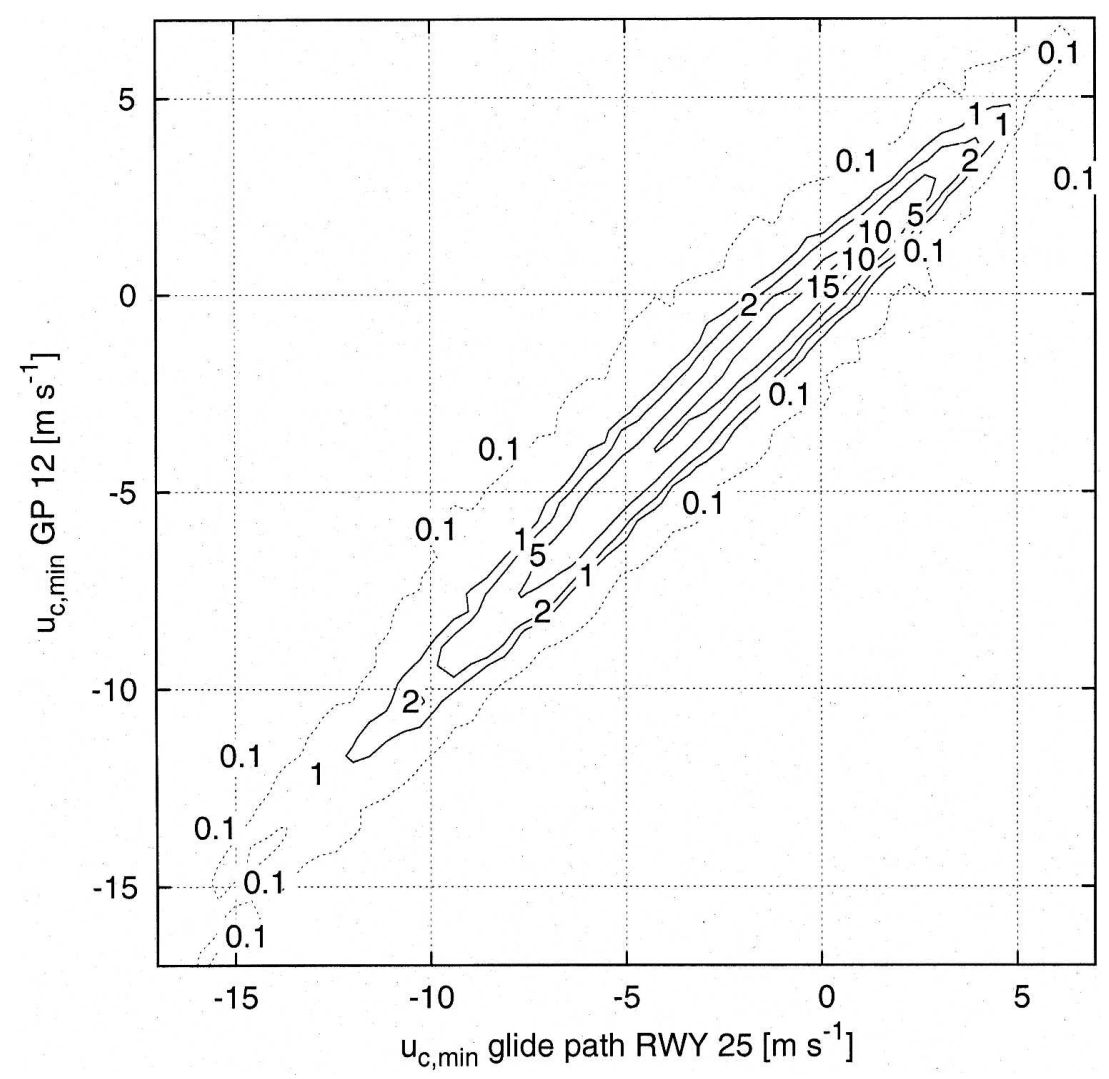

FIG. 18. Joint frequency distribution of minimum crosswind along the glide path to runway 25 vs minimum crosswind at grid point $12(\%)$. 


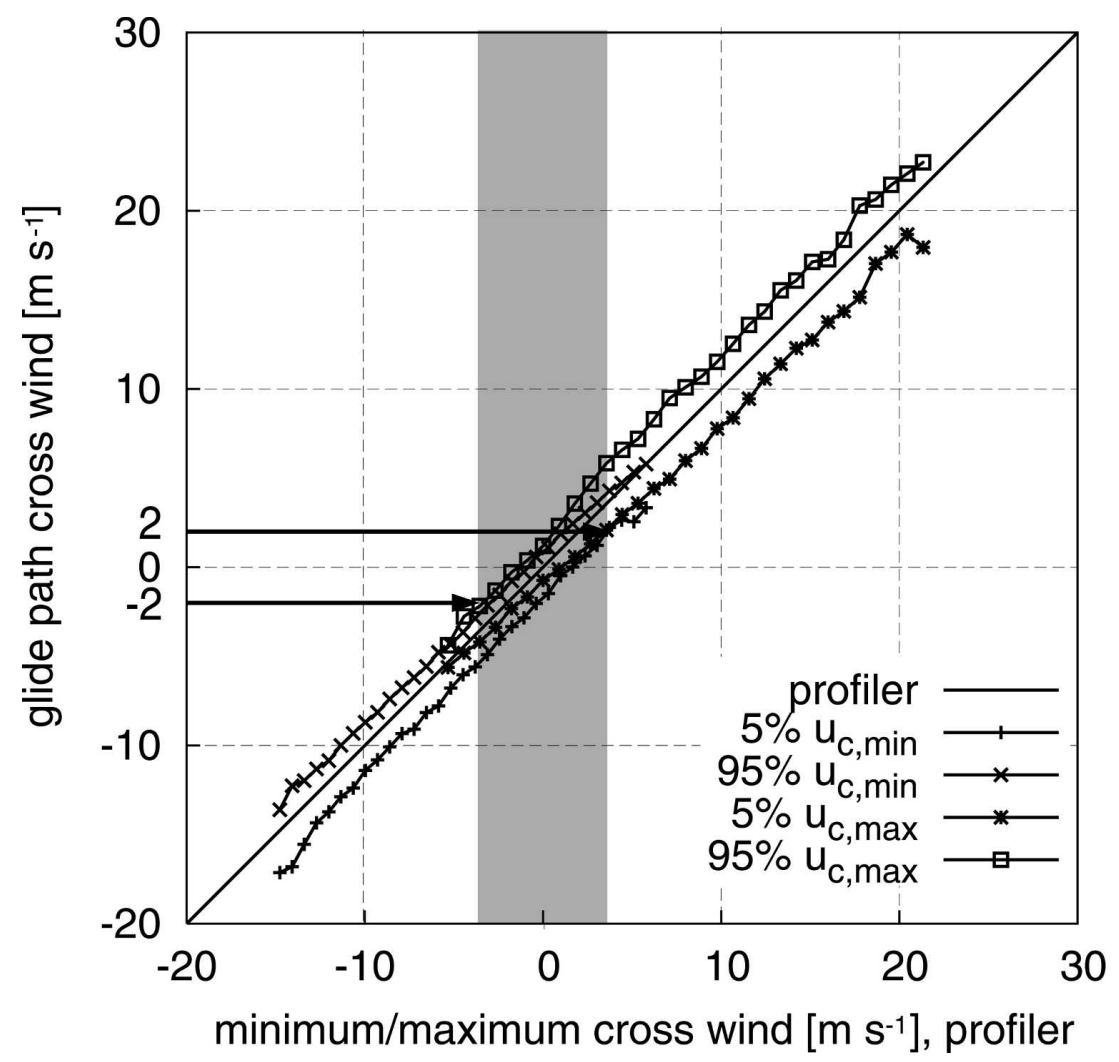

FIG. 19. Glide path variability as a function of the minimum and maximum crosswind measured by a hypothetical profiler. Safety allowances are based on the 5th and 95th percentile values of the deviations between minimum (maximum) profiler and glide path crosswind. The gray region denotes the crosswind interval of the profiler for which a crosswind of $\left|u_{c}\right|>2 \mathrm{~m} \mathrm{~s}^{-1}$ along the glide path cannot be expected. The safety allowances are computed from the 1-yr database.

we can parameterize the maximum crosswind as $u_{c, \max , 5}=0.92 u_{c, \text { max,profiler }}-1.0 \mathrm{~m} \mathrm{~s}^{-1}$ and $u_{c, \max , 95}=$ $1.01 u_{c, \text { max,profiler }}+1.64 \mathrm{~m} \mathrm{~s}^{-1}$. If we require that the $\mid u_{c}$, $\min \mid>2 \mathrm{~m} \mathrm{~s}^{-1}$, we can compute a crosswind threshold of $-3.6 \mathrm{~m} \mathrm{~s}^{-1}$ for the lower and $3.8 \mathrm{~m} \mathrm{~s}^{-1}$ for the upper bound for the wind profiler. Outside this interval we can expect sufficient large crosswind magnitudes all along the glide path, which, however, can be found only in $3 \%$ of profiles in the database. For the Frankfurt situation, this means that a wake avoidance system for a single runway approach, which solely relies on the crosswind using a wind profiler, will offer only very limited capacity potential. A similar result was found for parallel runway operations (Konopka and Fischer 2005).

The previous analysis evaluated the model-resolved spatial and temporal variability of the wind field. Unresolved turbulent fluctuations that are relevant for wake vortex transport have to be considered. This can be achieved in terms of an additional safety allowance as it is done in the probabilistic wake transport and decay model P2P (Holzäpfel 2003).

\section{Conclusions}

This paper provides a detailed analysis of the 1-yr meteorological database for the Frankfurt Airport area that has been generated by the model system NOWVIV. We have shown a good agreement between the joint frequency distribution of wind speed and direction in comparison with the 30-yr wind climatology. Observed differences may be attributed to the particular synoptic weather patterns of the year considered or to climate variability. We observe more frequent northeasterly wind directions relative to the long-term climatology. This appears to be a feature of climate variation and is supported by observations of ATC, who noticed more frequent landings on runway 07 in recent years.

A subset of 40 days from the weather database is validated against wind and temperature profiler data 
taken at Frankfurt Airport during autumn 2004. The joint frequency distributions of wind speed and direction at selected height levels show very good agreement with the wind profiler data revealing typical features of boundary layer wind profiles. Consequently, the frequency distributions of crosswind also agree very well with observations. Meteorological quantities that are of particular relevance for predicting wake vortex behavior, namely, crosswind, wind shear, eddy dissipation rate, and thermal stratification, are analyzed. Aside from eddy dissipation rate, the differences between predictions and observations are acceptably small. Larger differences are found close to the surface, which can be attributed to local surface features not resolved by the model. Close to the surface, the eddy dissipation rate extracted from the TKE budget equation of the weather prediction model MM5 is significantly overestimated relative to dissipation rates derived from lidar measurements. Above the atmospheric surface layer, the dissipation rate agrees well. We propose the use of a simple parameterization of dissipation rate in the surface layer, which has a reasonable agreement with observations.

The frequency distribution of stratification indicates an overestimation of very stable situations in comparison with observations. The absolute numbers of these very stable events, however, are small. In general, the model atmosphere tends to be well mixed, which can be seen by the large fraction of neutral/near-neutral stratification. These near-neutral stratified cases can be related to strong turbulence levels. The frequency distribution of wind shear agrees well with measurements.

We have analyzed the wind conditions along the glide path with a focus on wake avoidance due to lateral transport for a single runway approach. As an example, a crosswind threshold of $2 \mathrm{~m} \mathrm{~s}^{-1}$ was chosen, which is met in $12 \%$ of all glide path profiles in the database. A similar number is obtained if we consider only profiles at a grid point close to the airport, which suggests that a single wind profiler may be sufficient to describe the meteorological conditions along the glide path. Last, a simple parameterization for the model-resolved variability of crosswind along the glide path has been developed. The parameterization determines safety allowances that have to be added to the profiler crosswind. Unfortunately, the already small number of favorable meteorological conditions reduces from about $12 \%$ to $3 \%$. It can be concluded that crosswindbased wake avoidance for the single runway approach will provide only very little benefit in terms of a capacity gain for the given crosswind threshold using a wind profiler. The consideration of wake vortex decay and deformation together with vertical transport may help here, even though the complexity of such a wake vortex avoidance system increases substantially.

To sum up we conclude that the 1-yr database is representative of the conditions at Frankfurt Airport. The verification of the model results using measured profiles taken at the airport suggests that we can also expect realistic weather conditions along the glide path. Of course, a similar database may be easily generated for any other terminal area.

Acknowledgments. We gratefully acknowledge the excellent support and work of the teams from Airbus, DFS Deutsche Flugsicherung GmbH, DLR, Fraport AG, and METEK during the measurement campaign. The comments of the reviewers are greatly appreciated.

\section{REFERENCES}

Bergström, H., 1996: A climatological study of boundary layer wind speed using a meso- $\gamma$-scale higher-order closure model. J. Appl. Meteor., 35, 1291-1306.

Burk, S. D., and W. T. Thompson, 1989: A vertically nested regional numerical prediction model with second-order closure physics. Mon. Wea. Rev., 117, 2305-2324.

de Bruin, A. C., L. J. P. Speijker, H. Moet, B. Krag, R. Luckner, and S. Mason, 2003: S-WAKE: Assessment of wake vortex safety. National Aerospace Laboratory Tech. Rep., Publishable Summary Report NLR-TP-2003-243, 76 pp.

Donaldson, C., and A. Bilanin, 1975: Vortex wakes of conventional aircraft. AGARD Tech. Rep. 204, 79 pp.

Eurocontrol, 2001: ESSAR 4: Risk assessment and mitigation in ATM. EATMP Infocentre Eurocontrol Tech. Rep., 22 pp.

_ 2004: Challenges to growth. EATMP Infocentre Eurocontrol Tech. Rep., 66 pp.

Frech, M., 2006: Estimating the turbulent energy dissipation rate in an airport environment. Bound.-Layer Meteor., 123, 385393.

- , and T. Zinner, 2004: Concept of wake vortex behavior classes. J. Aircr., 41, 564-570.

— , F. Holzäpfel, T. Gerz, and J. Konopka, 2002: Short term prediction of the horizontal wind vector within a wake vortex warning system. Meteor. Appl., 9, 9-20.

Gerz, T., F. Holzäpfel, W. Bryant, F. Köpp, M. Frech, A. Tafferner, and G. Winckelmans, 2005: Research towards a wakevortex advisory system for optimal aircraft spacing. Compt. Rend. Phys., 6, 501-523.

Grell, G., J. Dudhia, and D. R. Stauffer, 1994: A description of the fifth-generation Penn State/NCAR Mesoscale Model (MM5). NCAR Tech. Note NCAR/TN-397+IA, 114 pp.

—, S. Emeis, W. R. Stockwell, T. Schoenemeyer, R. Forkel, J. Michalakes, R. Knoche, and W. Seidl, 2000: Application of a multiscale, coupled MM5/chemistry model to the complex terrain of the VOTALP valley campaign. Atmos. Environ., 34, 1435-1453.

Heimann, D., 1986: Estimation of regional surface layer wind field characteristics using a three layer mesoscale model. Beitr. Phys. Atmos., 59, 518-537.

Holzäpfel, F., 2003: Probabilistic two-phase wake vortex decay and transport model. J. Aircr., 40, 323-331.

, 2005: Probabilistic two-phase aircraft wake-vortex model: Further development and assessment. J. Aircr., 43, 700-708. 
—, and R. E. Robins, 2004: Probabilistic two-phase aircraft wake-vortex model: Application and assessment. J. Aircr., 41, 1117-1126.

— , T. Gerz, and R. Baumann, 2001: The turbulent decay of trailing vortex pairs in stably stratified environments. Aerosp. Sci. Technol., 5, 95-108.

Konopka, J., and H. Fischer, 2005: The wake vortex warning system at Frankfurt Airport. Proc. 24th Digital Avionics Systems Conf., Washington, DC, IEEE, 3.A.6-31-14.

Masson, V., J.-L. Champeaux, F. Chauvin, C. Meriguet, and R. Lacaze, 2003: A global database of land surface parameters at $1-\mathrm{km}$ resolution in meteorological and climate models. J. Climate, 16, 1261-1282.

Mengelkamp, H.-T., 1999: Wind climate simulations over complex terrain and wind turbine energy output estimation. Theor. Appl. Climatol., 63, 129-139.

Piper, M., 2001: The effects of a frontal passage on fine-scale nocturnal boundary layer turbulence. Ph.D. thesis, University of Colorado, 233 pp.
Sandström, S., 1997: Simulations of the climatological wind field in the Baltic Sea area using a mesoscale higher-order closure model. J. Appl. Meteor., 36, 1541-1552.

Sarpkaya, T., 2000: New model for vortex decay in the atmosphere. J. Aircr., 37, 53-61.

Smalikho, I., F. Köpp, and S. Rahm, 2005: Measurement of atmospheric turbulence by $2-\mu \mathrm{m}$ Doppler lidar. J. Atmos. Oceanic Technol., 22, 1733-1747.

Smirnova, T. G., J. M. Brown, S. G. Benjamin, and D. Kim, 2000: Parameterization of cold-season processes in the MAPS landsurface scheme. J. Geophys. Res., 105, 4077-4086.

Steppeler, J., G. Doms, U. Schättler, H. W. Bitzer, A. Gassmann, U. Damrath, and G. Gregoric, 2003: Meso-gamma scale forecast using the non-hydrostatic model LM. Meteor. Atmos. Phys., 82, 75-96.

Zhong, S., X. Hee-Jin, J. C. Bian, W. Heilman, and B. Potter, 2005: Evaluation of real-time high-resolution MM5 predictions over the Great Lakes region. Wea. Forecasting, 20, 6381. 